\title{
Hamiltonian formulation of systems with balanced loss-gain and exactly solvable models
}

\author{
Pijush K. Ghosh* and Debdeep Sinha ${ }^{\dagger}$ \\ Department of Physics, Siksha-Bhavana, \\ Visva-Bharati University, \\ Santiniketan, PIN 731 235, India.
}

\begin{abstract}
A Hamiltonian formulation of generic many-body systems with balanced loss and gain is presented. It is shown that a Hamiltonian formulation is possible only if the balancing of loss and gain terms occur in a pairwise fashion. It is also shown that with the choice of a suitable co-ordinate, the Hamiltonian can always be reformulated in the background of a pseudoEuclidean metric. If the equations of motion of some of the well-known many-body systems like Calogero models are generalized to include balanced loss and gain, it appears that the same may not be amenable to a Hamiltonian formulation. A few exactly solvable systems with balanced loss and gain, along with a set of integrals of motion is constructed. The examples include a coupled chain of nonlinear oscillators and a many-particle Calogero-type model with four-body inverse square plus two-body pair-wise harmonic interactions. For the case of nonlinear oscillators, stable solution exists even if the loss and gain parameter has unbounded upper range. Further, the range of the parameter for which the stable solutions are obtained is independent of the total number of the oscillators. The set of coupled nonlinear equations are solved exactly for the case when the values of all the constants of motions except the Hamiltonian are equal to zero. Exact, analytical classical solutions are presented for all the examples considered.
\end{abstract}

keywords: Hamiltonian formulation, Exactly solvable models, Dissipative system, Coupled nonlinear oscillators, Calogero-type model

\section{Contents}

1 Introduction

2 Hamiltonian Formulation 5

2.1 Representation of Matrices . . . . . . . . . . . . . . . . . 7

2.2 Examples . . . . . . . . . . . . . . . . . . . . . . . . 9

2.3 Rational Calogero $\operatorname{Model}(\mathrm{RCM}) \ldots \ldots \ldots \ldots \ldots \ldots \ldots$

*email: pijushkanti.ghosh@visva-bharati.ac.in

${ }^{\dagger}$ email: debdeepsinha.rs@visva-bharati.ac.in 
3 Exactly Solvable Models 13

3.1 Translationally invariant potential . . . . . . . . . . . . . . . 14

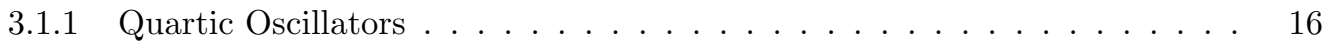

3.1.2 Embedded Rotational Symmetric System . . . . . . . . . . . . . . . 18

3.1.3 Calogero-type systems with four-body interaction . . . . . . . . . . 20

3.2 Rotationally invariant potential . . . . . . . . . . . . . . . . . . . . . . . . . . . . .

3.2.1 Exactly Solvable Models with $N=2 \ldots \ldots \ldots$. . . . . . . . . 23

3.2.2 Solvable Model of $N>2 \ldots \ldots \ldots \ldots$. . . . . . . . . . . . . . . . .

4 Summary \& Discussions 25

5 Acknowledgements 27

6 Appendix A 27

7 Appendix B 28

8 Appendix C $\quad 29$

9 Appendix D: $D_{N}$ type Calogero system with four-body interaction 30

\section{Introduction}

The Hamiltonian formulation of dissipative systems is a recurring theme in physics [1, 2]. One of the earliest approaches to the problem is to introduce an auxiliary system as a thermal bath that is time-reversed version of a dissipative harmonic oscillator. The original and the auxiliary oscillators considered together is described in terms of a Hamiltonian, when the loss and gain are balanced equally. The rates of dissipation from the system and absorption by bath are same and the total energy of the system-bath combination is conserved. Quantization of the Hamiltonian involves various subtle issues with important results and applications [3, 4, 5, 6, 7, 8, 9,

Studies on systems invariant under the combined operation of parity $(\mathcal{P})$ and time-reversal $(\mathcal{T})$ symmetry is currently an active area of research 10-14 with plethora of experimental results in the context of superconductivity [15, [16], optics [17]-20, microwave cavities [21, atomic diffusion 22, nuclear magnetic resonance 23], coupled electronic and mechanical oscillators 24, 20], coupled whispering galleries [25, 26] etc. It is within the context of experiments on coupled whispering gallery modes, the Hamiltonian of dissipative harmonic oscillators with balanced loss and gain was reconsidered. The Hamiltonian is invariant under combined $\mathcal{P} \mathcal{T}$-symmetry which the solutions do not obey. Consequently, the Hamiltonian neither admits classically stable solutions, nor quantum bound states. However, if the two oscillators are coupled through $\mathcal{P} \mathcal{T}$ symmetric interactions, classically stable solutions as well as quantum bound states exist within the ranges of the parameters for which $\mathcal{P} \mathcal{T}$ symmetry remains unbroken. An equilibrium is reached for the case of unbroken $\mathcal{P} \mathcal{T}$ symmetry so that the amount of energy transferred to the bath is reverted back to the system at the equal rate. This mathematical model explains well the results obtained in Ref. [26] and opens up several new possibilities.

Hamiltonians with balanced loss and gain describe a class of systems which are intermediate between open and closed systems. In the theory of quantum dissipation 27, 28, the system is coupled to bath consisting of infinitely many harmonic oscillators so that the energy can not be transferred back to the system. On the other hand, the sole objective of adding $\mathcal{P} \mathcal{T}$ symmetric 
interaction to the systems with balanced loss and gain is to achieve an equilibrium for which the energy is reverted back to the system from the bath at the same rate as it is deposited to the bath from the system. For the case of broken $\mathcal{P} \mathcal{T}$ symmetry, the equilibrium is lost and the Hamiltonian is suitable for describing open system.

Dissipation is a natural phenomenon and with the technological advancements, tailoring systems with balanced loss and gain is a reality. Within this background, it is utmost important to study more systems with balanced loss and gain. It may be recalled at this point that a system of coupled nonlinear oscillators with balanced loss and gain was investigated in Ref. 29] with some interesting results. In absence of any Hamiltonian formulation of this model, another system of nonlinear oscillators with balanced loss and gain was analyzed in Ref. [30] which admits a Hamiltonian formulation. A chain of linear oscillators with its continuum limit has been studied in Ref. [31. It has been shown recently that two-body rational Calogero model with balanced loss and gain terms is exactly solvable admitting classically stable solutions as well as quantum bound states in the unbroken $\mathcal{P} \mathcal{T}$ phase 32 . In spite of all of these developments, a Hamiltonian formulation of generic many-particle systems with balanced loss and gain in a model independent way is lacking. It may be noted that coupled non-linear Schrödinger equations with balanced loss and gain describe important physical effects in $\mathcal{P} \mathcal{T}$ symmetric theory. The Lagrangian and Hamiltonian formulation of such systems can be achieved in a straightforward way within the formulation of non-relativistic field theory. However, the issue of incorporating balanced loss and gain terms to generic many-particle classical and quantum mechanical systems has not been addressed in the literature with its full generality.

The purpose of this article is to present a systematic investigation on Hamiltonian formulation of generic many-particle classical and quantum mechanical systems with balanced loss and gain. The form of a generic many-particle Hamiltonian including gauge potentials is assumed and conditions are imposed so that the resulting equations of motion contain balanced loss and gain terms. This severely restricts the allowed ranges of gain-loss coefficients and types of interaction potentials. The balancing of loss and gain terms necessarily occurs in a pair-wise fashion, i.e. corresponding to the co-efficient $\gamma$ of a loss term, there necessarily exists a gain term with the co-efficient $-\gamma$. The balancing of $\gamma$ by two or more terms is strictly forbidden. Further, it appears that some of the well known many-particle systems like Calogero models [33, 34, 35, 36, 37] are not amenable to Hamiltonian formulation for three or more particles, if they are generalized to include balanced loss and gain terms. One important aspect of Calogero model is that each particle interacts with rest of the particles and it is manifested in the respective equations of motion. If this feature is relaxed, Hamiltonian formulation of many-body systems governed by inverse-square plus harmonic interaction with balanced loss and gain terms may be constructed. In fact, in spite of the imposing restrictions, a Hamiltonian formulation is possible for a very large class of systems with balanced loss and gain. The known examples are reproduced within the formulation presented in this article and some new examples are presented. Finally, it is shown that the Hamiltonian can always be reformulated in the background of a pseudo-Euclidean metric through some co-ordinate transformations. A Hamiltonian formulation for systems with spacedependent balanced loss and gain is also presented.

The systems with balanced loss and gain terms are investigated from the viewpoint of exactly solvable models. In particular, exactly solved systems are constructed for specified forms of potentials. Two classes of exactly solvable models with balanced loss and gain are presented which are characterized by invariance of the potential under specific symmetry transformations: (I) translation and (II) rotation in a pseudo-Euclidean space endowed with the metric $g_{i j}=$ $(-1)^{i+1} \delta_{i j}$ 38. It should be mentioned here that the action or the Hamiltonian of type-I systems is not invariant under translation, only the potential respects this symmetry. Similarly, the Hamiltonian for type-II models is not invariant under rotation in any plane in the pseudo- 
Euclidean metric, but, invariant under rotations in specific planes. The approach taken in constructing exactly solvable models for $N=2 m, m \in \mathbb{Z}^{+}$number of particles is the following. Apart from the Hamiltonian, $m$ number of integrals of motion are constructed for type-I as well as type-II models. These integrals of motion are in involution and imply that the system is at least partially integrable. The existence of these integrals of motion allows the dynamics of the original system with $2 m$ degrees of freedom to be determined in terms of an effective system with $m$ degrees of freedom. Consequently, the exactly solvability of the effective system ensures the same for the original Hamiltonian. This result is quite general and innumerable numbers of exactly solvable models with balanced loss and gain can be constructed.

Stable classical solutions are obtained in terms of Jacobi elliptic functions for particular ranges of parameters for the type-I models. For the case of a single quartic nonlinear oscillator, stable solutions are allowed even if the gain-loss parameter $\gamma$ is varied without any upper bound. It may be recalled at this point that for the case of linear oscillator 25, $\gamma$ can not exceed the value of the angular frequency of the harmonic oscillator for having stable solutions. The effect of the non-linearity is also significant for the case of coupled chain of nonlinear oscillators with uniform gain-loss parameters. Unlike the example considered in Ref. [31, the region in the parameter-space admitting stable solutions remains same for any number of particles. Exact classical solutions are obtained in closed analytical form for several models which are appropriate generalizations of well-known systems like, two and three particles coupled nonlinear oscillators, Henon-Heils system, Calogero-type models etc. The exact solutions of some of the many particle systems with balanced loss and gain and are interacting via a four-body potential are obtained. The exact solutions of these model are generated by exploiting the known solutions of Calogero type of models. It is found that the balancing the loss and gain term in a pair-wise fashion highly restricts the possible form of the four-body interaction.

Exact solutions can be found for several type-II models, including coupled chain of nonlinear oscillators. However, the solutions are not stable and there are no region in the parameter-space for which stable solutions are possible. There are equal number of growing and decaying solutions for even number of particles. Exact solutions are obtained in terms of Jacobi elliptic functions multiplied by an exponentially decaying/growing factor. It may be recalled that a system of harmonic oscillators with balanced loss and gain and without any coupling between the two has similar unstable solutions [1, 2]. The specified type of interaction allowed for type-II models do not lead to an equilibrium in respect of energy-transfer between the growing and the decaying modes and hence, the system is unstable.

The plan of the article is the following. The general Hamiltonian formulation of a generic system with balanced loss and gain is discussed in the next section. The imposing restrictions on possible forms of Hamiltonian are discussed in some detail in terms of properties of some matrices appearing in it. Possible representations of these matrices are discussed in Sec. 2.1. The condition for a known Hamiltonian to remain a Hamiltonian system, when it is generalized by including balanced loss and gain is discussed in Sec. 2.2. The condition is solved for a few cases and some previously studied systems are reproduced. The condition for Hamiltonian formulation of rational Calogero model with balanced loss and gain is given in Sec. 2.3 and solved for two particles. In absence of any solution of this condition for more than two particles, an example of a many-particle system with inverse-square plus harmonic interaction, which is different from rational Calogero model, is also presented. Exactly solvable models with stable solutions are presented in Sec. 3.1, while models with unstable solutions are presented in Sec. 3.2. Finally, in Sec. 4, the results are summarized and implications of the results as well as future directions are discussed. The results related to a few exactly solvable models are presented in Appendix-A and Appendix-B. The Lax-pair formulation of Sutherland is considered in Appendix-C and in Appendix-D, $D_{N}$-type Calogero model with four-body interaction is discussed. 


\section{Hamiltonian Formulation}

A systematic investigation on the Hamiltonian and Lagrangian formulation of many-particle systems with balanced loss and gain is presented in this section. The approach taken in this article is quite general and applicable to a large class of many-particle systems with balanced loss and gain. The Hamiltonian is assumed to be of the form,

$$
H=\Pi^{T} M \Pi+V\left(x_{1}, x_{2}, \ldots, x_{N}\right),
$$

where $X=\left(x_{1}, x_{2}, \ldots x_{N}\right)^{T}$ and $\Pi=\left(\pi_{1}, \pi_{2}, \ldots . \pi_{N}\right)^{T}$ are $N$ coordinates and their conjugate momenta, respectively. The suffix $T$ denotes transpose, i.e $X^{T}=\operatorname{Transpose}(X)$. The study of constrained systems is beyond the scope of the present article and hence, the $N \times N$ real symmetric matrix $M$ is taken to be non-singular. Any non-standard form of Hamiltonian is also excluded from the purview of present article. The generalized momenta $\Pi$ is defined as,

$$
\Pi=P+A X,
$$

where $P^{T}=\left(p_{1}, p_{2}, \ldots, p_{N}\right)$ is the conjugate momenta and $\mathrm{A}$ is a real $N \times N$ square matrix. Further restrictions on $M$ and $A$ will follow by demanding that $H$ describes a many-particle system with balanced loss and gain terms.

The Hamiltonian (11) may be re-expressed as,

$$
H=P^{T} M P+X^{T} A^{T} M P+P^{T} M A X+X^{T} A^{T} M A X+V\left(x_{1}, x_{2}, \ldots, x_{N}\right) .
$$

and the resulting Eqs. of motion has the following form:

$$
\begin{aligned}
& \ddot{X}-2 M R \dot{X}+2 M\left(\frac{\partial V}{\partial X}\right)=0 \\
& R:=A-A^{T}, \quad \frac{\partial V}{\partial X} \equiv\left(\frac{\partial V}{\partial x_{1}}, \frac{\partial V}{\partial x_{2}}, \ldots, \frac{\partial V}{\partial x_{N}}\right)^{T} .
\end{aligned}
$$

The matrix $A$ can be decomposed as the sum of a symmetric matrix $A_{s}$ and an anti-symmetric matrix $A_{a}$. The matrix $A_{s}$ does not contribute to the Eq. of motion (4). Further, $A_{s}$ can be gauged away from $\Pi$ in Eq. (2) and hence, from $H$ in the corresponding quantum theory. In particular,

$e^{-i X^{T} A_{s} X} \Pi e^{i X^{T} A_{s} X} \rightarrow \tilde{\Pi}:=P+A_{a} X=-i \frac{\partial}{\partial X}+A_{a} X, \quad \frac{\partial}{\partial X}:=\left(\frac{\partial}{\partial x_{1}}, \frac{\partial}{\partial x_{2}}, \ldots, \frac{\partial}{\partial x_{N}}\right)^{T}$,

where $\hbar=1$. Thus, the matrix $A$ can be chosen to be anti-symmetric without loss of any generality. The choice $A=\frac{R}{2}$ is consistent with the definition of anti-symmetric matrix $R$. The equation of motion in equation (4) may also be derived from the Lagrangian,

$$
\mathcal{L}=\frac{1}{4} \dot{X}^{T} M^{-1} \dot{X}+\frac{1}{4}\left(X^{T} R \dot{X}-\dot{X}^{T} R X\right)-V\left(x_{1}, x_{2}, \ldots, x_{N}\right),
$$

which is invariant under the operation of transposition, i.e., $\mathcal{L}^{T}=\mathcal{L}$, since $R$ is an anti-symmetric matrix by construction. The parity and time-reversal symmetry may be defined as,

$$
\mathcal{T}: t \rightarrow-t, \quad \mathcal{P}: X \rightarrow \tilde{X}=W X
$$

where $W$ is an $N \times N$ orthogonal matrix with determinant -1 . The invariance of the Lagrangian $\mathcal{L}$ and $H$ under $\mathcal{P} \mathcal{T}$ implies that $W$ commutes with $M$, while anti-commutes with $R$. 
A phase-space analysis of Eqs. (3) and (4) may be performed with the assumption that dissipation is due to terms linear in velocity in the equations of motion. The result shows that only the diagonal elements of the matrix $M R$ are relevant for determining whether or not the system is dissipative. The following condition is imposed by demanding that the velocity dependent term in the Eq. of motion for $x_{i}$ should only contain $\dot{x}_{i}$ :

$$
D=M R, \quad D:=\operatorname{diagonal}\left(\gamma_{1}, \gamma_{2}, \ldots . . \gamma_{N}\right), \gamma_{i} \in \Re .
$$

It can be shown that the matrices $M, R$ and $D$ anti-commute with each other:

$$
\{M, R\}=0, \quad\{R, D\}=0, \quad\{M, D\}=0 .
$$

The first relation is derived by using the fact that $M$ and $D$ are symmetric matrices, while $R$ is an anti-symmetric matrix. Rest of the relations follow from the first one and Eq. (8). All the above relations in Eq. (9) are true even if $D$ is non-diagonal, but a symmetric matrix. The anti-commutation relation involving $M$ and $D$ leads to trace less condition on $D$,

$$
\operatorname{Tr}(D)=0,
$$

implying that the gain and loss are balanced, i.e $\sum_{i} \gamma_{i}=0$. It can be further shown that the balancing of loss and gain terms necessarily occurs in a pairwise fashion for even $N$. In particular, if $\mathbf{v}$ is an eigen-vector of $D$ with non-vanishing eigen-value $\lambda$, then $M \mathbf{v}$ is also an eigen-vector of $D$ with eigenvalue $-\lambda$. Thus, the non-vanishing $\gamma_{i}$ 's are grouped into $N / 2$ independent pairs $\gamma_{i}+\gamma_{j}$ such that no two indices are repeated twice and each such pair is equal to zero. The anti-commutation relation between $M$ and $D$ also implies that at least one of the $\gamma_{i}$ 's must be equal to zero for odd $\mathrm{N}$ for which $\operatorname{det}(D)=0$ :

$$
\operatorname{det}(D)\left[1-(-1)^{N}\right]=0 .
$$

Further, it follows from Eq. (8) that $\operatorname{det}(R)=0$ for $\operatorname{det}(D)=0$, since $M$ is non-singular. Both $R$ and $D$ can be chosen to be non-singular for even $N$ and the following relations hold:

$$
\operatorname{Tr}(M)=0, \quad\left\{R^{-1}, D\right\}=0 .
$$

Further, for even $N$, the minimum and maximum numbers of independent non-vanishing offdiagonal elements of $M_{i j}$ are $N / 2$ and $N^{2} / 4$, respectively. The same result for odd $N$ may be obtained by substituting $N \rightarrow N-1$ in the respective expression for even $N$. For odd $N$, the element $M_{N N} \neq 0$.

A few comments are in order before proceeding further:

(1)The anti-commutation relation of $M$ with $R$ or $D$ for $N=2 m, m \in \mathbb{Z}^{+}$implies that corresponding to each of its $m$ positive eigenvalues $\lambda$, there exists an eigenvalue $-\lambda$. The diagonal matrix $M_{d}=\hat{O} M \hat{O}^{T}$ corresponding to $M$ has the expression $M_{d}=\operatorname{diagonal}\left(\lambda_{1},-\lambda_{1}, \lambda_{2},-\lambda_{2}\right.$, $\left.\ldots, \lambda_{m},-\lambda_{m}\right)$, where $\hat{O}$ is an orthogonal matrix and a particular ordering of eigenvalues is assumed. Any other ordering obtained by permuting the eigenvalues in the expression of $M_{d}$ is also acceptable. With the introduction of the anti-symmetric matrix $\tilde{R}=\hat{O} R \hat{O}^{T}$ and a set of new co-ordinates,

$$
\tilde{X}=\hat{O} X, \tilde{P}=\hat{O} P, \tilde{\Pi}=\hat{O} \Pi=\tilde{P}+\frac{1}{2} \tilde{R} \tilde{X},
$$

the Hamiltonian $H$ in Eq. (1) may be re-interpreted as defined in the background of an indefinite metric $M_{d}$ :

$$
H=\tilde{\Pi}^{T} M_{d} \tilde{\Pi}+V\left(\tilde{x}_{1}, \tilde{x}_{2}, \ldots, \tilde{x}_{N}\right)
$$




$$
=\tilde{P}^{T} M_{d} \tilde{P}+\tilde{X}^{T} Q \tilde{P}+\frac{1}{4} \tilde{X}^{T}\left(M_{d} \tilde{R}^{2}\right) \tilde{X}+V\left(\tilde{x}_{1}, \tilde{x}_{2}, \ldots, \tilde{x}_{N}\right),
$$

where $Q:=M_{d} \tilde{R}$ and $\left\{M_{d}, \tilde{R}\right\}=0$. The matrix $Q$ is symmetric with all of its diagonal elements equal to zero, while $M_{d} \tilde{R}^{2}$ is a symmetric matrix. It may be recalled that a similar indefinite metric appears in the Hamiltonian formulation of Pais-Uhlenbeck oscillator 39.

(2) Systems with space-dependent dissipation coefficient exhibit interesting physical effect and has various applications. Two common examples from this class of systems are van der pol oscillator [40] and van der Pol-Duffing oscillator [41]. The coefficient acts as a gain term in some region of space, while it is dissipative in some other region of space. Such a system may be generalized to include balanced loss and gain terms by considering a time-reversed version of it as a thermal bath with a Hamiltonian formulation for the system-bath combination. The loss term in some region of space for the system is equally balanced by a gain term for the bath in the same region of space and vice verse. The Hamiltonian is still given by (1) with $\Pi$ in Eq. (2) replaced by,

$$
\Pi=P+A F, \quad F \equiv\left(F_{1}\left(x_{1}, \ldots, x_{N}\right), F_{2}\left(x_{1}, \ldots, x_{N}\right), \ldots, F_{N}\left(x_{1}, \ldots, x_{N}\right)\right)^{T}
$$

where $F$ is a $N$ component column vector with each component depending on the dependent variables. A similar analysis as above shows that the equation of motion reads,

$$
\ddot{X}-2 Q D \dot{X}+2 M\left(\frac{\partial V}{\partial X}\right)=0, \quad Q \equiv \sum_{i=1}^{N} \frac{\partial F_{i}}{\partial x_{i}} .
$$

System with space-dependent gain-loss term is obtained for suitable choices of the function $F_{i}$. Note that when $F_{i}=\frac{x_{i}}{N}$, the system reduces to the form of system having constant loss and gain coefficient.

\subsection{Representation of Matrices}

The condition (8) can be realized for $N=2$ with the following choice:

$$
M=\sigma_{x}, \quad R=-i \gamma \sigma_{y}, \quad D=\gamma \sigma_{z},
$$

where $\gamma_{1}=-\gamma_{2} \equiv \gamma$ and $\sigma_{x}, \sigma_{y}, \sigma_{z}$ are Pauli spin matrices. Such a realization can be easily generalized to any even $N=2 m, m \in \mathbb{Z}^{+}$:

$$
M=\kappa \otimes \sigma_{x}, \quad D=\eta \otimes \sigma_{z}, \quad R=-i\left(\kappa^{-1} \eta\right) \otimes \sigma_{y},
$$

where the commutator of $m \times m$ diagonal matrix $\eta:=\operatorname{diagonal}\left(\gamma_{1}, \gamma_{3}, \ldots, \gamma_{m}\right)$ and the $m \times m$ invertible real symmetric matrix $\kappa$ must vanish, i.e.,

$$
[\kappa, \eta]=0 .
$$

The representations of $M$ and $D$ in Eq. (18) are chosen, while that of $R$ is determined by using the Eq. (8), i.e. $R=M^{-1} D$. The pair-wise balancing of loss and gain terms impose the conditions, $\gamma_{2 i}=-\gamma_{2 i-1}, i=1,2, \ldots m$. The constraint (19) can be solved in various ways. For example, $\eta=\gamma I_{m}$ solves Eq. (19) for any $\kappa$, where $I_{m}$ is $m \times m$ identity matrix. This choice corresponds to uniform gain and loss parameter $\gamma$, i.e. $\gamma_{2 i-1}=\gamma \forall i$. On the other hand, $\kappa=I_{m}$ solves Eq. (19) for any $\eta$ with $\gamma_{2 i}=-\gamma_{2 i-1}, i=1,2, \ldots m$. This corresponds to a system with non-uniform gain and loss parameters. 
It may be recalled that the Hamiltonian $H$ in Eq. (1) can be re-interpreted as defined in the background of an indefinite metric $M_{d}$. For the representation (18) with $\eta=\gamma I_{m}$ and $\kappa=I_{m}$, the diagonal matrix $M_{d}=\hat{O} M \hat{O}^{T}$ and the orthogonal matrix $\hat{O}$ has the following expressions:

$$
M_{d}=I_{m} \otimes \sigma_{z}, \quad \hat{O}=\frac{1}{\sqrt{2}}\left[I_{m} \otimes\left(\sigma_{x}+\sigma_{z}\right)\right] .
$$

The matrix $\hat{O}$ generates the new coordinates and momenta as defined by Eq. (13). The new Hamiltonian is given by Eq. (14) with

$$
Q=M_{d} \tilde{R}=\gamma I_{m} \otimes \sigma_{x}, \quad M_{d} \tilde{R}^{2}=-\gamma^{2} I_{m} \otimes \sigma_{z},
$$

and has the following form

$$
H=\tilde{P}^{T}\left[I_{m} \otimes \sigma_{z}\right] \tilde{P}+\gamma \tilde{X}^{T}\left[I_{m} \otimes \sigma_{x}\right] \tilde{P}-\frac{\gamma^{2}}{4} \tilde{X}^{T}\left[I_{m} \otimes \sigma_{z}\right] \tilde{X}+V\left(\tilde{x}_{1}, \tilde{x}_{2}, \ldots, \tilde{x}_{N}\right) .
$$

The first and third terms of Eq. (22) are quadratic in momenta and coordinate respectively and give the length in a pseudo Euclidean space. The second term is a sum of product of coordinates and momenta and bears the characteristic of balanced loss and gain system.

It may be noted that the representation (18) is not unique and there are several other possibilities. One such example is presented below:

$$
\begin{aligned}
& {[M]_{i j}=\delta_{i, j+1}+\delta_{i, j-1}, \quad[D]_{i j}=(-1)^{i+1} \gamma \delta_{i j}, \quad[R]_{i j}=(-1)^{j+1}\left[M^{-1}\right]_{i j},} \\
& {\left[M^{-1}\right]_{i j}=-\left[M^{-1}\right]_{j i}=\frac{1}{4}(-1)^{\frac{j-i-1}{2}}\left[1-(-1)^{j-i}\right]\left[1-(-1)^{i}\right], j>i .}
\end{aligned}
$$

The diagonal matrix $M_{d}=\hat{O} M \hat{O}^{T}$ corresponding to the representation (23), gives rise to a pseudo Euclidean metric where the form of the matrix $\hat{O}$, generating the new coordinates and momenta as defined by Eq. (13), is given by:

$$
\hat{O}_{i j}=\sqrt{\frac{2}{N+1}} \sin \left(\frac{i j \pi}{N+1}\right)
$$

and the form of $M_{d}$ becomes:

$$
M_{d}=\operatorname{diagonal}\left(2 \cos \frac{\pi}{N+1}, 2 \cos \frac{2 \pi}{N+1}, \ldots \ldots, 2 \cos \frac{N \pi}{N+1}\right) .
$$

It may be noted that the $i$-th and the $N+1-i$-th eigenvalues of $M_{d}$ are equal but opposite in sign. The new Hamiltonian is given by Eq. (14) with

$$
Q=M_{d} \tilde{R}=\gamma \delta_{i, N+1-i}, \quad M_{d} \tilde{R}^{2}=-\gamma^{2} M_{d}^{-1} .
$$

The Hamiltonian in the pseudo Euclidean space corresponding to the representations (18) and (23) are not equivalent in the sense that the determinant of $M_{d}$ is different in two cases. However, these two pseudo Euclidean metric can be made equivalent by simultaneous implication of the following rearrangements:

i) A proper scale transformation for the representation (23) of conjugate coordinates and momenta of the following form:

$$
\left(\tilde{p}_{k}, \tilde{p}_{N+1-k}\right) \rightarrow \sqrt{2 \cos \frac{k \pi}{N+1}}\left(\tilde{p}_{k}, \tilde{p}_{N+1-k}\right), \quad\left(\tilde{x}_{k}, \tilde{x}_{N+1-k}\right) \rightarrow \frac{1}{\sqrt{2 \cos \frac{k \pi}{N+1}}}\left(\tilde{x}_{k}, \tilde{x}_{N+1-k}\right)
$$




$$
k=1,2, \ldots, m \text {. }
$$

ii) In case of representation (18), a particular ordering of the eigenvalues of $M_{d}$ is assumed. If this ordering is made such that the $i$-th and the $N+1-i$-th eigenvalue become equal and opposite in sign.

Thus, for both the representations (18) and (23), a similarity transformation to a pseudo Euclidean space followed by a proper scaling of coordinates and momenta will give rise to the same form of the Hamiltonian and hence the same equations of motion. However, in original coordinates the representations (18) and (23) describe different systems having different form of potentials. The solutions of these different kind of systems can be found by exploiting the solutions obtained in a pseudo Euclidean space.

Either of the above representations of matrices for even $N=2 m$ can be suitably generalized for odd $N=2 m+1$. The $2 m+1 \times 2 m+1$ dimensional matrices $M, R, D$ are constructed by embedding the respective $2 m \times 2 m$ dimensional representation in it and choosing the extra matrix elements as follows:

$$
\begin{aligned}
& M_{2 m+1, i}=M_{i, 2 m+1}=0, i=1,2, \ldots 2 m, \quad M_{2 m+1,2 m+1} \neq 0, \\
& R_{i, 2 m+1}=R_{2 m+1, i}=0, D_{i, 2 m+1}=D_{2 m+1, i}=0, i=1,2, \ldots 2 m+1 .
\end{aligned}
$$

The equation governing the dynamics of $x_{2 m+1}$ does not contain any gain or loss term. However, it may be coupled to other degrees of freedom through appropriate choices of the interaction potential.

\subsection{Examples}

The Eq. of motion (4) can be re-written as,

$$
\ddot{X}-2 D \dot{X}+2 M\left(\frac{\partial V}{\partial X}\right)=0,
$$

and equivalently in component form:

$$
\ddot{x}_{i}-2 D_{i i} \dot{x}_{i}+2 \sum_{k=1}^{N} M_{i k} \frac{\partial V}{\partial x_{k}}=0, i=1,2, \ldots, N .
$$

Unlike in the standard Hamiltonian formulation, the Eq. of motion for $x_{i}$ does not contain the term $\frac{\partial V}{\partial x_{i}}$ for even $N$ due to the trace less condition on $M$. For odd $N$, only one diagonal element of $M$ may be chosen to be non-zero. Consequently, if $M_{p p} \neq 0$, the Eq. of motion for $x_{p}$ contains the term, $\frac{\partial V}{\partial x_{p}}$. If a system governed by the Hamiltonian $\tilde{H}=P^{T} P+\tilde{V}$ is generalized to include balanced loss and gain terms such that a Hamiltonian formulation in the form of $H$ is possible and the Eqs. of motion resulting from these two Hamiltonians are identical in the limit of vanishing loss and gain terms, then the following condition must hold:

$$
\frac{\partial V}{\partial x_{i}}=\sum_{k=1}^{N} M_{i k}^{-1} \frac{\partial \tilde{V}}{\partial x_{k}} i=1,2, \ldots, N
$$

This constitutes a set of coupled first-order differential equations. These equations are in general nonlinear, except for the case for which the potential $\tilde{V}$ is a quadratic form of the co-ordinates. The set of equations can be solved for quadratic $\tilde{V}$. In particular,

$$
\tilde{V}=X^{T} M G X \Longrightarrow V=X^{T} G X
$$


where $G$ is an arbitrary symmetric matrix. The system of coupled $\mathcal{P} \mathcal{T}$-symmetric oscillators with uniform loss and gain co-efficients and interaction parameters of Ref. 31 may be obtained by choosing the potential $G$ as,

$$
G=\omega^{2} M^{-1}+\epsilon I_{N},
$$

and using the representation of $M$ given in Eq. (23), where $I_{N}$ is $N \times N$ identity matrix. Similarly, the example of system with non-uniform parameters may also be reproduced with following choices of $M, D, R$ :

$$
[M]_{i j}=\delta_{j, N+1-i}, D=\delta_{i j} \gamma_{i}, \gamma_{N+1-i}=-\gamma_{i}, R=M^{-1} D=M D .
$$

The matrix $G$ for this case is an anti-tridiagonal matrix with the elements,

$$
G_{i j}=\omega_{i}^{2} \delta_{j, N+1-i}+\epsilon_{i}\left(\delta_{j, N-i}+\delta_{j, N+2-i}\right) .
$$

The formulation may be used to construct Hamiltonian system corresponding to coupled chain of nonlinear oscillators with balanced loss and gain terms. For example, a Hamiltonian system for two coupled nonlinear oscillators may be constructed by considering the representations of $M, R, D$ as in Eq. (17) and choosing the potential,

$$
V=a_{0} x_{1} x_{2}+a_{1}\left(x_{1}^{2}+x_{2}^{2}\right)+a_{2}\left(x_{1} x_{2}^{3}+x_{2} x_{1}^{3}\right)+a_{3} x_{1}^{2} x_{2}^{2}+a_{4}\left(x_{1}^{4}+x_{2}^{4}\right), a_{i} \in \Re .
$$

This system is $\mathcal{P} \mathcal{T}$-symmetric and reduces to the example studied in Ref. 30 for $a_{3}=0=a_{4}$. It is also evident that a Hamiltonian formulation of the system of nonlinear oscillators considered in Ref. [29] is forbidden. There are various possibilities for generalizing this system to $N$ coupled chain of nonlinear oscillators. A straightforward generalization would be to consider the potential,

$$
V=\sum_{i=1}^{N-1}\left[a_{0} x_{i} x_{i+1}+a_{2}\left(x_{i} x_{i+1}^{3}+x_{i+1} x_{i}^{3}\right)+a_{3} x_{i}^{2} x_{i+1}^{2}\right]+\sum_{i=1}^{N}\left[a_{1} x_{i}^{2}++a_{4} x_{i}^{4}\right], a_{i} \in \Re .
$$

and the representations in Eqs. (23) and (28). The parameters appearing in $V$ are chosen in such a way that the $H$ is $\mathcal{P} \mathcal{T}$-symmetric.

System with space-dependent dissipative coefficient can be obtained from Eq. (16). The example of this kind of systems are Van der pol oscillator [40, in case of which the dissipative term has the coefficient $\left(x^{2}-1\right)$, and duffing Van der pol oscillator 41. Oscillator systems having space dependent dissipative coefficient can easily be generated for the appropriate form of $F$ in Eq. (16). For example, in case of two coupled oscillators with space dependent balanced loss and gain terms, the form of $F$ may be considered as $F_{i}=\frac{1}{2} x_{i}-\frac{1}{3} x_{i}^{3}$ which gives $Q=\left[1-\left(x_{1}^{2}+x_{2}^{2}\right)\right]$. The various type of coupling between the two oscillators can be generated by choosing appropriate form of the potential $V$. In this case Eq. (16) takes the following form:

$$
\begin{aligned}
& \ddot{x}_{1}-2 \gamma\left[1-\left(x_{1}^{2}+x_{2}^{2}\right)\right] \dot{x}_{1}+\frac{\partial V}{\partial x_{2}}=0, \\
& \ddot{x}_{2}+2 \gamma\left[1-\left(x_{1}^{2}+x_{2}^{2}\right)\right] \dot{x}_{2}+\frac{\partial V}{\partial x_{1}}=0,
\end{aligned}
$$

where $M, R, D$ are as in Eq. (17). It may be noted that inside the circle $x_{1}^{2}+x_{2}^{2}=1$, the first oscillator has a gain and the second oscillator has an equal amount of loss and out side the circle the situation is reversed. 


\subsection{Rational Calogero Model(RCM)}

Given the importance of RCM and its relation to various diverse areas of physics, it is desirable to find its generalization involving balanced loss and gain terms. The potential $\tilde{V}_{R C M}$ for the RCM has the following form:

$$
\tilde{V}_{R C M}=\frac{\omega^{2}}{2} \sum_{i=1}^{N} x_{i}^{2}+\sum_{\substack{i, j=1 \\ i<j}}^{N} \frac{g}{\left(x_{i}-x_{j}\right)^{2}} .
$$

An important feature of RCM is that each particle interacts with rest of the particles through inverse-square interaction. The potential $V_{R C M}$ is determined through a set of $N$ coupled firstorder nonlinear partial differential equations:

$$
\frac{\partial V_{R C M}}{\partial x_{i}}=\sum_{k=1}^{N} M_{i k}^{-1}\left[\omega^{2} x_{k}-\sum_{\substack{l=1 \\ l \neq k}}^{N} \frac{2 g}{\left(x_{k}-x_{l}\right)^{3}}\right] .
$$

For $N=2, V_{R C M}$ can be determined easily:

$$
V_{R C M}=\omega^{2} x_{1} x_{2}-\frac{g}{\left(x_{1}-x_{2}\right)^{2}},
$$

where the representation of $M$ is given by (17). The classical as well as the quantum version of this two-body rational Calogero model has been studied in detail in Ref. [32]. Finding a solution for $N>2$ and $g \neq 0$ is a highly nontrivial problem and it seems that a consistent solution of Eq. (41) may not exist. Many-body systems with balanced loss and gain terms may be constructed, where particles interact through harmonic plus inverse-square interaction. However, in the limit of vanishing loss/gain parameters, the equations of motion differ from that of RCM. These systems may be identified as Calogero-type models with balanced loss and gain terms. Such a system may be obtained easily by substituting $\tilde{V}_{R C M}$ for $V$ in Eqs. (113229130). The resulting Eqs. of motion with the representation of $M$ as in Eq. (18) read:

$$
\begin{aligned}
& \ddot{x}_{1}-2 \gamma \dot{x}_{1}+2 \omega^{2} x_{2}-\sum_{\substack{k=1 \\
k \neq 2}}^{N} \frac{4 g}{\left(x_{2}-x_{k}\right)^{3}}=0 \\
& \ddot{x}_{i}-2 \gamma(-1)^{i+1} \dot{x}_{i}+2 \omega^{2}\left(x_{i-1}+x_{i+1}\right)-\sum_{\substack{k=1 \\
k \neq i-1}}^{N} \frac{4 g}{\left(x_{i-1}-x_{k}\right)^{3}}-\sum_{\substack{k=1 \\
k \neq i+1}}^{N} \frac{4 g}{\left(x_{i+1}-x_{k}\right)^{3}}=0, \\
& 2 \leq i \leq N-1, \\
& \ddot{x}_{N}-2 \gamma(-1)^{N+1} \dot{x}_{N}+2 \omega^{2} x_{N-1}-\sum_{\substack{k=1 \\
k \neq N-1}}^{N} \frac{4 g}{\left(x_{N-1}-x_{k}\right)^{3}}=0 .
\end{aligned}
$$

The Eqs. of motion of classical RCM is not reproduced in the limit of vanishing $\gamma$. It may be noted that in Eq. (29), the column matrix $\frac{\partial V}{\partial X}$ is multiplied by the off diagonal matrix $M$ which hinder $N$ particle generalization of RCM with balanced loss and gain. In the case of RCM, the dynamics governing the $i$ 'th particle contains interaction terms where the $i$ 'th particle interacts through pair-wise harmonic plus inverse-square interaction with all other particles. However, in the present case, the dynamics governing the $i$ 'th particle contains interaction terms where its 
nearest neighbors interact with all other particles including it through pair-wise harmonic plus inverse-square interaction. In the limit of vanishing $g$, the coupled chain of linear oscillators considered in Ref. [31] is reproduced. Thus, the Hamiltonian which gives rise to Eqs. (43) is an appropriate generalization of the system considered in Ref. 31] with inverse-square interaction. This constitutes a new class of models which deserves further investigations. The exact solvability and/or integrability of the system is not apparent and beyond the scope of the investigations of the present article.

The most general integrable potential for Calogero-Moser-Sutherland(CMS) system is the Weierstrass elliptic potential of the following form $V(x)=\mathcal{P}\left(x, 2 \omega_{1}, 2 \omega_{2}\right)$. This is a single valued doubly periodic function of a single complex variable $x$ with $\omega_{1}$ and $\omega_{2}$ are being the two half periods. In the limit $\omega_{1} \rightarrow \infty, \omega_{2} \rightarrow \infty, V(x) \rightarrow x^{-2}$ and we get the rational CMS model 33 . The trigonometric or hyperbolic CMS model arises when either $\omega_{1}$ or $\omega_{2} \rightarrow \infty$. Below we discuss the case of trigonometric and hyperbolic potentials with balanced loss and gain.

The Sutherland model was originally motivated to extract thermodynamics from the model having only the rational interaction term. The Calogero model with only the rational potential is not a bound system and one could put the system in a finite periodic box, such that the particles interact through all the infinitely many periodic images of themselves. In this case the two-body potential becomes a periodic one. The Sutherland model can easily be generalized to incorporate the balanced loss and gain term for two particles system. For three or more particles, the Hamiltonian formulation is possible in the presence of balanced loss and gain term only if we relax the pair-wise interaction among the particles. The Sutherland model with trigonometric interaction is given by the potential:

$$
\tilde{V}_{S}=\sum_{\substack{i, j=1 \\ i<j}}^{N} \frac{a^{2}}{\sin ^{2} a\left(x_{i}-x_{j}\right)} .
$$

The potential $V_{S}$ can be determined from Eq. (31):

$$
\frac{\partial V_{S}}{\partial x_{i}}=\sum_{k=1}^{N} M_{i k}^{-1}\left[\sum_{\substack{l=1 \\ k>l}}^{N} \frac{4 a^{3} \cos a\left(x_{k}-x_{l}\right)}{\sin ^{2} a\left(x_{k}-x_{l}\right)}-\sum_{\substack{l=1 \\ k<l}}^{N} \frac{4 a^{3} \cos a\left(x_{k}-x_{l}\right)}{\sin ^{2} a\left(x_{k}-x_{l}\right)} .\right] .
$$

This is a set of $N$ coupled first order nonlinear partial differential equations. For $N=2, V_{S}$ can be determined easily:

$$
V_{S}=-\frac{a^{2}}{\sin ^{2} a\left(x_{i}-x_{j}\right)},
$$

where the representation of $M$ is given by (17). For two particles case, the vanishing loss and gain parameter will produce the original model. The solutions of this model with balancing loss and gain term allow stable solutions. This model will be considered in detail in Ref. [42]. For three or more particles the solutions of Eq. (45) is highly nontrivial and it seems that a consistent solution may not exist. A Hamiltonian formulation for many particle system having balanced loss and gain and are interacting through a trigonometric potential may be constructed. However, in this case the vanishing loss and gain parameter will not produce the original model. This kind of model may be obtained by replacing $\tilde{V}_{S}$ by $V_{S}$ in constructing the Eqs of motion. The resulting Eqs. of motion are given by:

$$
\ddot{x}_{1}-2 \gamma \dot{x}_{1}+\sum_{\substack{l=1 \\ 2>l}}^{N} \frac{8 a^{3} \cos a\left(x_{2}-x_{l}\right)}{\sin ^{2} a\left(x_{2}-x_{l}\right)}-\sum_{\substack{l=1 \\ 2<l}}^{N} \frac{8 a^{3} \cos a\left(x_{2}-x_{l}\right)}{\sin ^{2} a\left(x_{2}-x_{l}\right)}=0,
$$




$$
\begin{aligned}
& \ddot{x}_{i}-2 \gamma(-1)^{i+1} \dot{x}_{i}+\sum_{\substack{l=1 \\
i-1>l}}^{N} \frac{8 a^{3} \cos a\left(x_{i-1}-x_{l}\right)}{\sin ^{2} a\left(x_{i-1}-x_{l}\right)}-\sum_{\substack{l=1 \\
i-1<l}}^{N} \frac{8 a^{3} \cos a\left(x_{i-1}-x_{l}\right)}{\sin ^{2} a\left(x_{i-1}-x_{l}\right)}, \\
& +\sum_{\substack{l=1 \\
i+1>l}}^{N} \frac{8 a^{3} \cos a\left(x_{i+1}-x_{l}\right)}{\sin ^{2} a\left(x_{i+1}-x_{l}\right)}-\sum_{\substack{l=1 \\
i+1<l}}^{N} \frac{8 a^{3} \cos a\left(x_{i+1}-x_{l}\right)}{\sin ^{2} a\left(x_{i+1}-x_{l}\right)}=0 \\
& 2 \leq i \leq N-1, \\
& \ddot{x}_{N}-2 \gamma(-1)^{N+1} \dot{x}_{N}+\sum_{\substack{l=1 \\
N-1>l}}^{N} \frac{8 a^{3} \cos a\left(x_{N-1}-x_{l}\right)}{\sin ^{2} a\left(x_{N-1}-x_{l}\right)} \\
& -\sum_{\substack{l=1 \\
N-1<l}}^{N} \frac{8 a^{3} \cos a\left(x_{N-1}-x_{l}\right)}{\sin ^{2} a\left(x_{N-1}-x_{l}\right)}=0
\end{aligned}
$$

where the representation of $M$ is given by (18). The above model describes a system where the dynamics of the $i$-th particle involves interacting term where its nearest neighbor interact with all other particles including it. This constitutes a new class of model and needs further investigations. A similar construction for hyperbolic potential is possible in the presence of balanced loss and gain term if we replace $a$ by $i a$ in all above calculations.

The many particle interaction of Calogero model is governed by root system of finite reflection groups. The type of Calogero model, we consider in this work belongs to A-type root system. However, Calogero-Moser system is integrable for other classical root systems (B,C,D) [35] as well for exceptional and non-crystallographic root systems [43]. For two particles case, various root systems can be modified to incorporate the loss and gain terms. Detail analysis regarding these models will be considered in [42]. However, all these models are not amenable to Hamiltonian formulation for three or more particles in the sense that the systems are not reduced to the original many-particle model in the limit of vanishing loss/gain parameter.

\section{Exactly Solvable Models}

The Hamiltonian is a constant of motion. The invariance of the Lagrangian $\mathcal{L}$ under different symmetry transformations may lead to other first integrals of the system. Invariance under spacetranslation is ruled out even for translationally invariant potential $V$. This is due to the presence of terms linear in $X$ and $X^{T}$, i.e. the second and the third terms in Eq. (6). Similarly, invariance under rotation is also ruled out even for rotationally invariant potentials. Nevertheless, integrals of motion may be constructed for specific choices of $M, D, R$ and $V$. A few such exactly solvable many-particle systems with $N=2 m, m \in \mathbf{Z}^{+}$are presented in this section.

For the representation (18) with $\eta=\gamma I_{m}$ and $\kappa=I_{m}$, a co-ordinate transformation of the form (13) by the matrix $\hat{O}$ as given by Eq. (20) with $\tilde{X}=\left(z_{i}^{+}, z_{i}^{-}\right)^{T}, i=1,2, \ldots \ldots, m$ and $X=\left(x_{2 i-1}, z_{2 i}\right)^{T}, i=1,2, \ldots \ldots, m$, generates the following set of new co-ordinates:

$$
z_{i}^{-}=\frac{1}{\sqrt{2}}\left(x_{2 i-1}-x_{2 i}\right), \quad z_{i}^{+}=\frac{1}{\sqrt{2}}\left(x_{2 i-1}+x_{2 i}\right), i=1,2, \ldots m
$$

Writing Eq. (22) in the component form, the Hamiltonian is obtained in the background of a pseudo Euclidean metric in the following form:

$$
H=\sum_{i=1}^{m}\left[\left(P_{z_{i}^{+}}^{2}-P_{z_{i}^{-}}^{2}\right)+\gamma\left(z_{i}^{+} P_{z_{i}^{-}}+z_{i}^{-} P_{z_{i}^{+}}\right)-\frac{\gamma^{2}}{4}\left\{\left(z_{i}^{+}\right)^{2}-\left(z_{i}^{-}\right)^{2}\right\}\right]+V\left(z_{i}^{+}, z_{i}^{-}\right)
$$


The conjugate momenta have the following expressions:

$$
P_{z_{i}^{+}}=\frac{1}{2}\left(\dot{z}_{i}^{+}-\gamma z_{i}^{-}\right), \quad P_{z_{i}^{-}}=-\frac{1}{2}\left(\dot{z}_{i}^{-}-\gamma z_{i}^{+}\right) .
$$

The Eqs. of motion can be derived from the Hamilton's equations of motion as,

$$
\ddot{z}_{i}^{+}-2 \gamma \dot{z}_{i}^{-}+2 \frac{\partial V}{\partial z_{i}^{+}}=0, \quad \ddot{z}_{i}^{-}-2 \gamma \dot{z}_{i}^{+}-2 \frac{\partial V}{\partial z_{i}^{-}}=0, \quad i=1,2, \ldots m .
$$

In the subsequent discussions $2 V$ is replaced by $V$. The Eqs. (51) is a set of $2 m$ coupled differential equations.

The parity $(\mathcal{P})$ transformation is defined as,

$$
\begin{aligned}
\mathcal{P}: \quad & X \rightarrow-\left(I_{m} \otimes \sigma_{x}\right) X, P \rightarrow-\left(I_{m} \otimes \sigma_{x}\right) P \\
& z_{i}^{+} \rightarrow-z_{i}^{+}, \quad z_{i}^{-} \rightarrow z_{i}^{-}, P_{z_{i}^{+}} \rightarrow-P_{z_{i}^{+}}, \quad P_{z_{i}^{-}} \rightarrow P_{z_{i}^{-}} .
\end{aligned}
$$

For odd $N=2 m+1$, the above relations are supplemented with the transformations for $x_{2 m+1}$ and $p_{2 m+1}, x_{2 m+1} \rightarrow x_{2 m+1}, p_{2 m+1} \rightarrow p_{2 m+1}$. The time-reversal transformation $\mathcal{T}$ is defined as,

$$
\mathcal{T}: X \rightarrow X, P \rightarrow-P, \Longrightarrow z_{i}^{+} \rightarrow z_{i}^{+}, z_{i}^{-} \rightarrow z_{i}^{-} \quad P_{z_{i}^{+}} \rightarrow-P_{z_{i}^{+}}, P_{z_{i}^{-}} \rightarrow-P_{z_{i}^{-}} .
$$

For odd number of particles $N=2 m+1$, the above relations are supplemented with the relations: $x_{2 m+1} \rightarrow x_{2 m+1}, p_{2 m+1} \rightarrow-p_{2 m+1}$. The Hamiltonian is invariant under the combined operation of $\mathcal{P} \mathcal{T}$ provided the real potential $V$ satisfies the condition:

$$
V\left(z_{i}^{+}, z_{i}^{-}\right)=V\left(-z_{i}^{+}, z_{i}^{-}\right) .
$$

It may be noted that the parity transformation defined by Eq. (52) is not unique. Consequently, the condition on $V\left(z_{i}^{-}, z_{i}^{+}\right)$to be $\mathcal{P} \mathcal{T}$ symmetric also varies depending on the choice of $\mathcal{P}$. For example, the parity transformation $\mathcal{P}_{1}$

$$
\mathcal{P}_{1}: z_{i}^{+} \rightarrow z_{i}^{+}, \quad z_{i}^{-} \rightarrow-z_{i}^{-}, P_{z_{i}^{+}} \rightarrow P_{z_{i}^{+}}, \quad P_{z_{i}^{-}} \rightarrow-P_{z_{i}^{-}},
$$

keeps $H$ in Eq. (49) $\mathcal{P}_{1} \mathcal{T}$ invariant provided $V\left(z_{i}^{+}, z_{i}^{-}\right)=V\left(z_{i}^{+},-z_{i}^{-}\right)$. Exactly solvable models can be found for several specific choices of $V$ satisfying the above constraints. A few examples with $\mathcal{P} \mathcal{T}$ invariance and the condition (54) are presented below.

\subsection{Translationally invariant potential}

The set of $2 m$ equations (51) can be decoupled into $m$ coupled equations for the choices of $V \equiv$ $V\left(z_{i}^{-}\right)$or $V \equiv V\left(z_{i}^{+}\right)$. The potential is translationally invariant for $V \equiv V\left(z_{i}^{-}\right)$. In particular, the potential remains invariant under the transformations $x_{2 i-1} \rightarrow x_{2 i-1}+\eta_{i}, x_{2 i} \rightarrow x_{2 i}+\eta_{i}$, where $\eta_{i}$ 's are $m$ independent parameters. The form of the potential is special in the sense that it allows $m$ independent parameters $\eta_{i}$ instead of a single one. The Hamiltonian is translationally invariant. The potential is chosen to be independent of $z_{i}^{+}$co-ordinates and hence, $V$ as well as $H$ is $\mathcal{P} \mathcal{T}$ symmetric. With the choice of this translationally invariant potential, the first set of Eqs. of (51) can be solved as,

$$
\dot{z}_{i}^{+}=2 \gamma z_{i}^{-}+\Pi_{i}, \quad \Pi_{i} \in \Re \forall i
$$


which when substituted in the second set of Eqs. result in the following set of $m$ coupled differential equations:

$$
\ddot{z}_{i}^{-}-4 \gamma^{2} z_{i}^{-}-\frac{\partial V}{\partial z_{i}^{-}}=2 \gamma \Pi_{i}, \quad i=1,2, \ldots m .
$$

where $\Pi_{i}$ 's are $m$ integration constants to be fixed by imposing initial conditions. The coordinates $z_{i}^{+}$are determined as,

$$
z_{i}^{+}(t)=2 \gamma \int z_{i}^{-}(t) d t+\Pi_{i} t+C_{i}, \quad C_{i} \in \Re \forall i,
$$

where $C_{i}$ 's are integration constants. The inhomogeneous term on the right hand side of Eq. (57) poses problem for finding exact solutions for a large class of $V$ and can be absorbed through a constant shift of the co-ordinates,

$$
z_{i}^{-} \rightarrow z_{i}=z_{i}^{-}+\frac{\Pi_{i}}{2 \gamma}
$$

which reduces Eqs. (57) and (58) to the following forms:

$$
\begin{aligned}
& \ddot{z}_{i}-4 \gamma^{2} z_{i}-\frac{\partial V\left(z_{i}\right)}{\partial z_{i}}=0, \\
& z_{i}^{+}(t)=2 \gamma \int z_{i}(t) d t+C_{i}, \quad i=1,2, \ldots m .
\end{aligned}
$$

However, an unpleasant outcome of this transformation is that the potential $V$, in general, becomes dependent on the dissipation parameter $\gamma$. In order to avoid such undesirable feature of the potential, the inhomogeneous part on the right hand side of Eq. (57) is taken to be zero by appropriate choice of initial conditions. In particular, the initial conditions on $\dot{z}_{i}^{+}(0)$ and $z_{i}^{-}(0)$ :

$$
\dot{z}_{i}^{+}(0)=2 \gamma z_{i}^{-}(0), \forall i
$$

gives the values of $m$ integration constants $\Pi_{i}=0$. Initial conditions on $z_{i}^{+}(0)$ and $\dot{z}_{i}^{-}(0)$ may be chosen independently and are to be specified later. It may be noted that $z_{i}^{-}=z_{i}$ for $\Pi_{i}=0$ and Eqs. (60) are considered for further discussions.

The $\Pi_{i}$ 's are $m$ integrals of motion:

$$
\Pi_{i}=2 P_{z_{i}^{+}}-\gamma z_{i}^{-},\left\{\Pi_{i}, \Pi_{j}\right\}_{P B}=0, \quad\left\{H, \Pi_{i}\right\}_{P B}=0,
$$

where $\{,\}_{P B}$ denotes the Poisson bracket. The Hamiltonian along with $\Pi_{i}$ 's constitute $m+1$ integrals of motion, implying that the system is at least partially integrable. It may be noted that the existence of $m$ integrals of motion is a consequence of the invariance of the Hamiltonian under translations with $m$ independent parameters $\eta_{i}$. If the potential is chosen as, $V \equiv V\left(z_{i}^{+}\right)$, then $H$ is invariant under translations $z_{i}^{-} \rightarrow z_{i}^{-}+\eta_{i}$. The corresponding conserved quantities $\Pi_{i}^{-}=2 P_{z_{i}^{-}}+\gamma z_{i}^{+}$are in involution and constitute $m$ integrals of motion implying partial integrability of the system. The form of the potential for both the cases discussed above needs to be explicitly specified in order to explore admissible exactly solvable models. The dynamical equations satisfied by $z_{i}^{-}$and $z_{i}^{+}$for $V \equiv V\left(z_{i}^{+}\right)$and $V \equiv V\left(z_{i}^{-}\right)$are related to each through the duality relation:

$$
z_{i}^{-} \leftrightarrow z_{i}^{+}, \quad V\left(z_{i}^{-}\right) \leftrightarrow-V\left(z_{i}^{+}\right), \quad \Pi_{i} \leftrightarrow-\Pi_{i}^{-} .
$$

In particular, equation satisfied by $z_{i}^{-}$and $z_{i}^{+}$for $V \equiv V\left(z_{i}^{+}\right)$may be obtained from Eqs. (57) and (58) by using the above transformation and without any change for $t, C_{i}$. Further investigations on exactly solvable models will be restricted to the case $V \equiv V\left(z_{i}^{-}\right)$in this article. The duality relation may be used to obtain results for $V \equiv V\left(z_{i}^{+}\right)$. There are plenty of choices of $V$ for which system governed by Eq. (60) is exactly solvable. 


\subsubsection{Quartic Oscillators}

One of the simplest choices for $m=1, N=2$ is to consider the case of cubic nonlinear oscillator:

$$
\begin{aligned}
& V\left(z_{1}\right)=-2 \omega_{0}^{2} z_{1}^{2}-\frac{\alpha}{4} z_{1}^{4}, \omega, \alpha \in \Re, \\
& \ddot{z}_{1}+\omega^{2} z_{1}+\alpha z_{1}^{3}, \omega^{2} \equiv 4\left(\omega_{0}^{2}-\gamma^{2}\right) .
\end{aligned}
$$

There are three different parametric regions for which exact solutions can be obtained in terms of Jacobi Elliptic functions:

(i) $\omega^{2}>0, \alpha>0$ : The gain-loss parameter $\gamma$ is restricted to lie in the range, $-\omega_{0}<\gamma<\omega_{0}$.

$$
\begin{aligned}
& z_{1}(t)=A c n(\Omega t, k), \quad z_{1}^{+}(t)=\frac{2 \gamma}{\Omega} \frac{\cos ^{-1}\{d n(\Omega t, k)\} \operatorname{sn}(\Omega t, k)}{\sqrt{1-d n^{2}(\Omega t, k)}} \\
& \Omega=\sqrt{\omega^{2}+\alpha A^{2}}, k^{2}=\frac{\alpha A^{2}}{2 \Omega^{2}},
\end{aligned}
$$

Non-singular stable solutions are obtained for $0<k<1$.

(ii) $\omega^{2}>0, \alpha<0$ : The gain-loss parameter $\gamma$ is restricted to lie in the range, $-\omega_{0}<\gamma<\omega_{0}$.

$$
\begin{aligned}
& z_{1}(t)=A \operatorname{sn}(\Omega t, k), z_{1}^{+}(t)=\frac{2 \gamma}{\sqrt{k} \Omega} \log [\operatorname{dn}(\Omega t, k)-\sqrt{k} c n(\Omega t, k)] \\
& \Omega=\left(\omega^{2}-\frac{|\alpha| A^{2}}{2}\right)^{\frac{1}{2}}, k^{2}=\frac{|\alpha| A^{2}}{2 \Omega^{2}}, 0 \leq A \leq \sqrt{\frac{\omega^{2}}{|\alpha|}}
\end{aligned}
$$

The restriction for having non-singular solution is $0<k<1$.

(iii) $\omega^{2}<0, \alpha>0$ : Unlike the previous two cases, the angular frequency is restricted to lie in the range, $-\gamma<\omega_{0}<\gamma$. The solutions are unstable for the case of linear oscillators with balanced loss and gain 25, whenever the gain-loss parameter $\gamma$ exceeds the value of angular frequency. However, the effect of the nonlinear term is to allow stable solutions even when $\gamma>\omega_{0}$. The stable solutions exists, even if the confining harmonic term is absent, i.e., $\omega_{0}=0$. There is no restriction on the possible range of $\gamma$ for this particular choice of potential. In particular, there are two different types of solutions depending on the ranges of amplitude $A$ of the solution of $z_{1}(t)$, one of which is stable and bounded. The unstable solution has the form:

$$
\begin{aligned}
& z_{1}(t)=\operatorname{Adn}(\Omega t, k), \quad z_{1}^{+}(t)=\frac{2 \gamma}{\Omega} \operatorname{am}(t, k) \\
& \Omega=\left(\frac{\alpha A^{2}}{2}\right), k^{2}=\frac{\alpha A^{2}-\left|\omega^{2}\right|}{2 \Omega^{2}}, \sqrt{\frac{\left|\omega^{2}\right|}{\alpha}} \leq A \leq \sqrt{\frac{2\left|\omega^{2}\right|}{\alpha}}
\end{aligned}
$$

where $z_{1}^{+}(t)$ is unbounded within the range $0<k<1$. The second solution is obtained for $\sqrt{\frac{2\left|\omega^{2}\right|}{\alpha}} \leq A<\infty$ and is similar to Eq. (65) except for the expressions for $\Omega$ and $k$. The non-singular, stable solution is obtained as,

$$
\begin{aligned}
& z_{1}(t)=A \operatorname{cn}(\Omega t, k), \quad z_{1}^{+}(t)=\frac{2 \gamma}{\Omega} \frac{\cos ^{-1}\{d n(\Omega t, k)\} \operatorname{sn}(\Omega t, k)}{\sqrt{1-d n^{2}(\Omega t, k)}} \\
& \Omega=\sqrt{-\left|\omega^{2}\right|+\alpha A^{2}}, \quad k^{2}=\frac{\alpha A^{2}}{2 \Omega^{2}} .
\end{aligned}
$$


The effect of the non-linear interaction is significant, it allows an unbounded upper range for $\gamma$.

A natural choice for $m=2, N=4$ is to consider the potential for a coupled quartic nonlinear oscillators:

$$
V=\sum_{i=1}^{2}\left[-2 \omega_{i}^{2} z_{i}^{2}-\frac{\alpha_{i}}{4} z_{i}^{4}\right]-\beta z_{1}^{2} z_{2}^{2}
$$

There are four distinct regions in parameter-space for which exact solutions exist 44]. For example, for the choice of the parameters $\omega_{1}=\omega_{2} \equiv \omega_{0}, \alpha_{1}=\alpha_{2} \equiv \alpha, 2 \beta=3 \alpha, z_{i}$ satisfy the equation:

$$
\ddot{z}_{1}+\omega^{2} z_{1}+\alpha\left(z_{1}^{3}+3 z_{1} z_{2}^{2}\right)=0, \ddot{z}_{2}+\omega^{2} z_{2}+\alpha\left(z_{2}^{3}+3 z_{1}^{2} z_{2}\right)=0, \omega^{2}=4\left(\omega_{0}^{2}-\gamma^{2}\right) .
$$

These two equations are separable in the co-ordinate, $u=z_{1}+z_{2}, v=z_{1}-z_{2}$ and describe two cubic oscillators:

$$
\ddot{u}+\omega^{2} u+\alpha u^{3}=0, \ddot{v}+\omega^{2} v+\alpha v^{3}=0 .
$$

As discussed for the case of $m=1, N=2$, there are three distinct regions in the parameter-space for which exact solutions exist for a cubic oscillators. In each region,the solutions for $z_{1}(t)$ and $z_{2}(t)$ may be constructed by combining $u \equiv u\left(A_{1}, k_{1}\right)$ and $v \equiv v\left(A_{2}, k_{2}\right)$ for different values of the amplitude $A$ and the modulus $k$. In particular,

$$
z_{1}(t)=\frac{1}{2}\left[u\left(A_{1}, k_{1}\right)+v\left(A_{2}, k_{2}\right)\right], z_{2}(t)=\frac{1}{2}\left[u\left(A_{1}, k_{1}\right)-v\left(A_{2}, k_{2}\right)\right] .
$$

For example, we may consider the solution in the range $\omega>0, \alpha>0$. In this range the solutions for $u$ and $v$ are given as:

$$
u_{i}=A_{i} c n\left(\Omega_{i} t, k_{i}\right), \quad \Omega_{i}=\sqrt{\omega^{2}+\alpha A_{i}^{2}}, \quad k_{1}^{2}=\frac{\alpha A_{1}^{2}}{2 \Omega_{1}^{2}}, \quad i=1,2, \quad u_{1}=u, u_{2}=v
$$

Therefore, we have:

$$
\begin{aligned}
& z_{1}(t)=A_{1} c n\left(\Omega_{1} t, k_{1}\right)+A_{2} c n\left(\Omega_{1} t, k_{2}\right) \\
& z_{2}(t)=A_{1} c n\left(\Omega_{1} t, k_{1}\right)-A_{2} c n\left(\Omega_{1} t, k_{2}\right)
\end{aligned}
$$

and

$$
z_{1}^{+}=\sum_{i=1}^{2} E\left(A_{i}, k_{i}\right), \quad z_{2}^{+}=\sum_{i=1}^{2}(-1)^{i+1} E\left(A_{i}, k_{i}\right)
$$

where

$$
E\left(A_{i}, k_{i}\right)=2 \gamma\left[\frac{\cos ^{-1}\left\{d n\left(\Omega_{i} t, k_{i}\right)\right\} \operatorname{sn}\left(\Omega_{i} t, k_{i}\right)}{\Omega_{i} \sqrt{1-d n^{2}\left(\Omega_{i} t, k_{i}\right)}}\right] .
$$

In terms of $x_{i}, i=1,2,3,4$ variables the solutions are:

$$
\begin{aligned}
& x_{1}=\sum_{i=1}^{2} \frac{1}{\sqrt{2}}\left[E\left(A_{i}, k_{i}\right)+A_{i} \operatorname{cn}\left(\Omega_{i} t, k_{i}\right)\right], \quad x_{2}=\sum_{i=1}^{2} \frac{1}{\sqrt{2}}\left[E\left(A_{i}, k_{i}\right)-A_{i} \operatorname{cn}\left(\Omega_{i} t, k_{i}\right)\right], \\
& x_{3}=\sum_{i=1}^{2}(-1)^{i+1} \frac{1}{\sqrt{2}}\left[E\left(A_{i}, k_{i}\right)+A_{i} \operatorname{cn}\left(\Omega_{i} t, k_{i}\right)\right],
\end{aligned}
$$




$$
x_{4}=\sum_{i=1}^{2}(-1)^{i+1} \frac{1}{\sqrt{2}}\left[E\left(A_{i}, k_{i}\right)-A_{i} \operatorname{cn}\left(\Omega_{i} t, k_{i}\right)\right],
$$

Non-singular stable solutions are obtained for $0<k_{1}<1$ and $0<k_{2}<1$. Solutions for the other range of the parameters are obtained similarly by using the Eqs. (66) and (67). For the choice of the parameters $\omega_{i}=\omega, \alpha_{i}=\alpha$ and $\beta=0$, the potential in Eq. (69) becomes spherically symmetric. This case will be considered in the next section where spherically symmetric systems are discussed.

\subsubsection{Embedded Rotational Symmetric System}

The original problem involving $2 m$ particles has been reduced to the study of a $m$ particle sub-system in terms of coordinates $z_{i}$. Rotationally symmetric potential in this $m$ dimensional subspace is considered. A new variable is introduced,

$$
r^{2}=\sum_{i=1}^{m} z_{i}^{2}
$$

which may be identified as a radial variable in $m$ dimensional Euclidean sub-space spanned by $z_{i}$ co-ordinates. The $z_{i}$ co-ordinates satisfy the equation,

$$
\ddot{z}_{i}-4 \gamma^{2} z_{i}-\frac{1}{r} \frac{\partial V}{\partial r} z_{i}=0
$$

for rotationally symmetric potential $V(r)$. This equation can be expressed solely in terms of radial variable in $m$ dimensional hyper-spherical co-ordinate,

$$
\ddot{r}-4 \gamma^{2} r-\frac{L^{2}}{r^{3}}-\frac{\partial V}{\partial r}=0,
$$

where $L^{2}$ is the square of the angular momentum and a constant of motion. Exact solutions may be obtained for specific choices of $V(r)$. The potential is chosen as,

$$
V(r)=-2 \omega_{0}^{2} r^{2}-\frac{\alpha}{4} r^{4}-\frac{\delta}{2 r^{2}}, \alpha, \delta \in \Re,
$$

for which Eq. (80) reduces to the form:

$$
\ddot{r}+\omega^{2} r+\left(\alpha r^{3}-\frac{L^{2}-\delta}{r^{3}}\right)=0, \quad \omega^{2} \equiv 4\left(\omega_{0}^{2}-\gamma^{2}\right) .
$$

Three different parametric choices of the potential are discussed below separately:

(a) $\alpha=\delta=0$ : This describes a trivial generalization of coupled dissipative oscillator model considered in Ref. 25]. In fact, the system governed by the Hamiltonian (49) for this case is $\mathrm{m}$ copies of the system considered in Ref. 25. with $\epsilon=-\omega_{0}^{2}$. Each of the $m$ uncoupled subsystems describes a Hamiltonian of two oscillators with balanced loss and gain. A shift of the co-ordinates $z_{i}^{-}$by an amount $\frac{2 \gamma \Pi_{i}}{4 \omega^{2}}$ casts the governing equations of motion as $m$ decoupled harmonic oscillators, which are exactly solvable.

(b) $\alpha \neq 0, \delta \neq 0$ : In this case the Eq. (83) becomes:

$$
\ddot{r}+\omega^{2} r+\left(\alpha r^{3}-\frac{L^{2}-\delta}{r^{3}}\right)=0,
$$


The solution of which may be written as:

$$
\begin{gathered}
r(t)=\left[\alpha_{3}-\left(\alpha_{3}-\alpha_{2}\right) s n^{2}(\lambda t, k)\right]^{\frac{1}{2}}, 0<k^{2}=\frac{\alpha_{3}-\alpha_{2}}{\alpha_{3}-\alpha_{1}}<1, \\
\lambda^{2}=\frac{\alpha}{2}\left(\alpha_{3}-\alpha_{1}\right),
\end{gathered}
$$

where $\alpha_{1}, \alpha_{2}, \alpha_{3}$ are constants satisfying the conditions:

$$
\begin{aligned}
& \alpha_{1}+\alpha_{2}+\alpha_{3}=-\frac{2 \omega^{2}}{\alpha}, \quad \alpha_{1} \alpha_{2} \alpha_{3}=-\frac{2\left(L^{2}-\delta\right)}{\alpha}, \\
& \alpha_{1} \alpha_{2}+\alpha_{2} \alpha_{3}+\alpha_{3} \alpha_{1}=\frac{4 E}{\alpha} .
\end{aligned}
$$

Further, Eq. (85) implies that for real $r, \alpha_{2}$ must be positive and the restriction on $k$ implies $\alpha_{2}>\alpha_{3}, \alpha_{1}$ and $E$ is the energy corresponding to $m$ oscillators. The angular momentum $L$ is a constant of motion. In general the solution for the angular part is a bit involved and is discussed in Appendix-B. However, a simplified solution for the angular variables may be obtained if we take the angular momentum to be identically zero for which all the angular variables become constant. With this simplifying assumption, all the $z_{i}$ are easily determined and we have:

$$
z_{i}=\left[\alpha_{3}-\left(\alpha_{3}-\alpha_{2}\right) s n^{2}(\lambda t, k)\right]^{\frac{1}{2}} f_{i}\left(\theta_{i}\right)
$$

where $f_{i}\left(\theta_{i}\right)$ is the function of angular coordinates of the form appears in the hyper-spherical coordinate system and is a constant in this case. The solutions of $z_{i}^{+}$are of the form

$$
z_{i}^{+}=2 \gamma f_{i}\left(\theta_{i}\right) I(t)
$$

with

$$
\begin{gathered}
I(t)=-\frac{i\left(\alpha_{3} w^{2} \operatorname{EllipF}(l(t), m)+\left(\alpha_{3}-\alpha_{2}\right) \operatorname{EllipP} i\left(\frac{1}{w^{2}}, l(t), m\right)\right) n(t)}{w \lambda d c(\lambda t, k)\left[\alpha_{3}-\left(\alpha_{3}-\alpha_{2}\right) s n^{2}(\lambda t, k)\right]^{\frac{1}{2}}}, \\
l(t)=i \sinh ^{-1}(w s c[\lambda t, k]), \quad w^{2}=\frac{\alpha_{2}}{\alpha_{3}}, \quad 0<m=\frac{w^{2}}{(1-k)}<1, \\
n(t)=\left[\left(1+w^{2} s c^{2}[\lambda t, k]\right)\left(1-k \operatorname{sn}^{2}[\lambda t, k]\right)\right]^{\frac{1}{2}} .
\end{gathered}
$$

It should be mentioned here that the expression of $I(t)$ encounters singularity and therefore the solutions of $z_{i}^{+}$'s are not stable.

(c) $\delta=0$ : The system reduces to a chain of coupled nonlinear oscillators with non-linearity arising due to cubic terms. This may be considered as the rotationally symmetric case of Eq. (71) in $m$ dimension. The Hamiltonian which produces Eq. (83) is known to be integrable for several distinct choices of the parameters 44. For $\delta=0$ and $m=2$, Eq. (84) describes a quartic oscillator and the solution for $r$ takes the following form:

$$
r(t)=\left[\frac{2}{\alpha}\left(E+\omega^{2}-\sqrt{\frac{\alpha}{2} \omega}\right)\right]^{\frac{1}{2}} \sin \left(\frac{\alpha}{2}(t+c)\right)
$$

where $c$ is a constant of integration and $z_{1}, z_{2}$ are respectively given as:

$$
z_{1}=\left[\frac{2}{\alpha}\left(E+\omega^{2}-\sqrt{\frac{\alpha}{2} \omega}\right)\right]^{\frac{1}{2}} \sin \left(\frac{\alpha}{2}(t+c)\right) \cos \theta_{1},
$$




$$
z_{2}=\left[\frac{2}{\alpha}\left(E+\omega^{2}-\sqrt{\frac{\alpha}{2} \omega}\right)\right]^{\frac{1}{2}} \sin \left(\frac{\alpha}{2}(t+c)\right) \sin \theta_{1}
$$

and the form of $z_{1}^{+}$and $z_{2}^{+}$are obtained as:

$$
\begin{aligned}
& z_{1}^{+}=-2 \gamma\left(\frac{\alpha}{2}\left[\frac{2}{\alpha}\left(E+\omega^{2}-\sqrt{\frac{\alpha}{2} \omega}\right)\right]^{\frac{1}{2}} \cos \left(\frac{\alpha}{2}(t+c)\right) \cos \theta_{1},\right. \\
& z_{2}^{+}=-2 \gamma\left(\frac{\alpha}{2}\left[\frac{2}{\alpha}\left(E+\omega^{2}-\sqrt{\frac{\alpha}{2} \omega}\right)\right]^{\frac{1}{2}} \cos \left(\frac{\alpha}{2}(t+c)\right) \sin \theta_{1} .\right.
\end{aligned}
$$

It should be mentioned here that the region of stable solution in the parameter-space remains same for any number of particles which is markedly different to the example considered in Ref. [31] where the stability region disappears as the number of oscillators is increased. The solutions for $r$ and angular variables $\theta_{i}, i=1,2, \ldots, m-1$ for nonzero $L$ are discussed in the Appendix B.

\subsubsection{Calogero-type systems with four-body interaction}

In this subsection a solvable many particle model with four-body interaction in one dimension in the presence of balanced loss and gain terms is investigated. Many-body systems with fourbody interaction have been considered earlier in the literature [45, 46, 47]. The exactly solvable quantum models of Calogero and Sutherland type with translationally invariant two and fourbody interactions is investigated in Ref. 46. An exactly solvable four-body interaction with non-translationally invariant interactions is discussed in Ref. [47. In our example, the four-body inverse square interaction is generated in the presence of balanced loss and gain terms in the original coordinate $x_{i}$ by considering a Calogero-type of potential for the reduced system in $z_{i}$ coordinates. The exact known solutions of Calogero-type system is then exploited to obtain the exact solutions of the four-body model.

The Calogero-type of system arises if we take the potential $V\left(z_{i}\right)$ as

$$
V\left(z_{i}\right)=-\sum_{i}^{m} 2 \omega_{0}^{2} z_{i}^{2}-\sum_{\substack{i, j=1 \\ i<j}}^{m} \frac{g^{2}}{2\left(z_{i}-z_{j}\right)^{2}}
$$

and the Eq. of motion (60) becomes:

$$
\begin{aligned}
& \ddot{z}_{i}+\omega^{2} z_{i}-\sum_{j,(j \neq i)}^{m} \frac{g^{2}}{\left(z_{i}-z_{j}\right)^{3}}=0 \\
& z_{i}^{+}(t)=2 \gamma \int z_{i}(t) d t+C_{i}, \quad i=1,2, \ldots m .
\end{aligned}
$$

The four-body interaction manifests itself in the potential as well as in the equations of motion, when $z_{i}$ 's are replaced by the original coordinates $x_{i}$. In particular, the potential

$$
V=-\sum_{i}^{m} \omega_{0}^{2}\left(x_{2 i-1}-x_{2 i}\right)^{2}-\sum_{\substack{i, j=1 \\ i<j}}^{m} \frac{g^{2}}{\left(x_{2 i-1}-x_{2 i}-x_{2 j-1}+x_{2 j}\right)^{2}},
$$

describes a pair-wise two-body harmonic term plus a four-body inverse square interaction. The equations of motion are,

$$
\ddot{x}_{2 l-1}-2 \gamma \dot{x}_{2 l-1}+2 \omega_{0}^{2}\left(x_{2 l-1}-x_{2 l}\right)-\sum_{\substack{i=1 \\ i \neq l}}^{m} \frac{2 g^{2}}{\left(x_{2 i-1}-x_{2 i}-x_{2 l-1}+x_{2 l}\right)^{3}}=0,
$$




$$
\ddot{x}_{2 l}+2 \gamma \dot{x}_{2 l}-2 \omega_{0}^{2}\left(x_{2 l-1}-x_{2 l}\right)+\sum_{\substack{i=1 \\ i \neq l}}^{m} \frac{2 g^{2}}{\left(x_{2 i-1}-x_{2 i}-x_{2 l-1}+x_{2 l}\right)^{3}}=0, l=1, . . m .(10
$$

Note that the permutation symmetry of the reduced system in terms of $z_{i}$ is lost when expressed in terms of the original coordinates $x_{i}$.

The classical solutions of Eq. (99) are the well known solutions of rational Calogero system with a harmonic confinement and the solutions for $z_{i}^{+}(t)$ can be obtained by integrating Eq. (100). The solution of Eq. (99) may be obtained from the Lax-pair formulation 48] (see Appendix-C), and is given by the eigenvalue of the following matrix:

$$
Q(t)=Q(0) \cos (\omega t)+\omega^{-1} \dot{Q}(0) \sin (\omega t),
$$

where $Q(0)=X(0)$ and $\dot{Q}(0)=L(0)$ are obtained from the initial values of $z_{i}(0)$ and $p_{z_{i}}(0)$ (for the expression of the matrices $\mathrm{Q}, \mathrm{L}, \mathcal{M}$, see Appendix-C). The expressions for $z_{i}^{+}$'s are given by the eigenvalues of the matrix $R(t)$, where

$$
R(t)=2 \gamma\left(\frac{X(0)}{\omega} \sin (\omega t)-\omega^{-2} L(0) \cos (\omega t)\right) .
$$

The closed form expressions for the eigenvalues of $Q(t)$ and $R(t)$ can be obtained easily for small $N$.

The case $m=1, N=2$ describes a system with only two-body interaction and is discussed in detail in Ref. 32. The four-body interaction appears in the Hamiltonian for $N \geq 4$. For the case $N=4$, the eigenvalues of $Q(t)$ gives:

$$
\begin{aligned}
& z_{1}=\frac{1}{2}\left[\left(a_{1}+a_{2}\right)+\sqrt{\left(a_{1}-a_{2}\right)^{2}+b^{2}}\right] \\
& z_{2}=\frac{1}{2}\left[\left(a_{1}+a_{2}\right)-\sqrt{\left(a_{1}-a_{2}\right)^{2}+b^{2}}\right]
\end{aligned}
$$

where

$$
\begin{aligned}
a_{j} & =z_{j}(0) \cos \omega t+\frac{p_{z_{j}}(0) \sin \omega t}{\omega}, j=1,2 \\
b^{2} & =\frac{g^{2} \sin ^{2} \omega t}{\left(z_{1}(0)-z_{2}(0)\right)^{2}} .
\end{aligned}
$$

and from Eq. (104), the eigenvalues of $R(t)$ may be written as

$$
\begin{aligned}
& z_{1}^{+}=\frac{\gamma}{\omega}\left[\left(c_{1}+c_{2}\right)+\sqrt{\left(c_{1}-c_{2}\right)^{2}+d^{2}}\right] \\
& z_{2}^{+}=\frac{\gamma}{\omega}\left[\left(c_{1}+c_{2}\right)-\sqrt{\left(c_{1}-c_{2}\right)^{2}+d^{2}}\right]
\end{aligned}
$$

where

$$
\begin{aligned}
c_{j} & =z_{j}(0) \sin \omega t-\frac{p_{z_{j}}(0) \cos \omega t}{\omega}, j=1,2 \\
d^{2} & =\frac{g^{2} \cos ^{2} \omega t}{\left(z_{1}(0)-z_{2}(0)\right)^{2}} .
\end{aligned}
$$

Now $x_{i}, i=1,2,3,4$ can easily be determined from Eq. (48). The solutions are periodic and stable. Solutions for $N>4$ may be obtained in a similar way. 
A few comments are in order before concluding the section;

i) The most general four-body inverse-square interaction for a many-particle system is of the form 45]:

$$
V_{4}=\sum_{\substack{i, j, p, q=1 \\ i \neq j \neq p \neq q}}^{N} \frac{g}{\left(x_{i}-x_{j}-x_{p}+x_{q}\right)^{2}}
$$

which is invariant under permutation symmetry. On the other hand, the potential in Eq. (101) lacks permutation symmetry. It is interesting to note that the inverse-square part of the potential in Eq. (101), i.e.

$$
\tilde{V}_{4}=\sum_{\substack{i, j=1 \\ i<j}}^{m} \frac{g^{2}}{\left(x_{2 i-1}-x_{2 i}-x_{2 j-1}+x_{2 j}\right)^{2}}
$$

forms a subset of the set of all the terms in (113). Further, although the form of $\tilde{V}_{4}$ changes with a change in the representations of $M, D$ and $R$, it always generates terms which form a subset of terms presented in $V_{4}$.

ii) It should be mentioned here that another four-body exactly solvable model may be generated in a similar way if we consider the Sutherland model for the reduced system in $z_{i}$ coordinate. However, in this case in order to remove the harmonic term proportional to $4 \gamma^{2}$ a counter term should be included in the potential, i. e., the potential should be of the form:

$$
V\left(z_{i}\right)=-\sum_{i}^{m} 2 \gamma^{2} z_{i}^{2}-\sum_{\substack{i, j=1 \\ i<j}}^{m} \frac{g^{2}}{\sin ^{2} g\left(z_{i}-z_{j}\right)} .
$$

This kind of potential gives Sutherland model for the reduced system in $z_{i}$ coordinates whose classical solutions are well known 48. These solutions may be exploited to generate the solutions of a system having trigonometric four-body interaction. The solution of Calogero model for any root system with four-body interaction can be obtained in a similar manner. For an example $D_{N}$ type Calogero model with four-body interaction is carried out in Appendix-C.

\subsection{Rotationally invariant potential}

A constant of motion may be constructed for a class of potential $V$ :

$$
\begin{aligned}
\hat{L} & =\frac{1}{2}\left(P^{T} D X+X^{T} D P\right) \\
& =\tilde{X}^{T} Q \tilde{P}
\end{aligned}
$$

which can be interpreted as angular momentum in a co-ordinate system endowed with the metric $M_{d}$ for specific forms of $R$. The time-variation of $\hat{L}$ is related to the potential $V$,

$$
\frac{d \hat{L}}{d t}=-X^{T} D \frac{\partial V}{\partial X}
$$

and $\hat{L}$ is a constant of motion provided the following condition is satisfied:

$$
X^{T} D \frac{\partial V}{\partial X}=0
$$


The constraint (118) may be solved with the following ansatz for $V$ :

$$
V \equiv V(r), \quad r^{2} \equiv X^{T} G X, \quad\{G, D\}=0,
$$

where $G$ is a symmetric matrix. Both $M$ and its inverse $M^{-1}$ are symmetric and anti-commute with $D$. For $N=2$, with the representation of the matrices $M, R, D$ given by Eq. (17), $G$ is uniquely fixed to be $G=M=\sigma_{x}$. For $N>2$, one may choose $G=a M+b M^{-1}, a, b \in \Re$. However, this choice is not unique, there are several other possibilities. For example, the matrices $M, R . D$ may be chosen from a real representation of Clifford algebra 49 for $N=2^{m}, m \in \mathbb{Z}^{+}$. It is always possible to find a representation where the number of non-diagonal symmetric matrices is $m$. Thus, for $N>2, G$ and $M$ can be chosen independently.

\subsubsection{Exactly Solvable Models with $N=2$}

The existence of two first integrals $H$ and $\hat{L}$ implies that the governing system is integrable for $N=2$ for any $V(r)$. The representations of $M, R, D$ are given by Eq. (17) and the matrix $G=M=\sigma_{x}$ and $r=\sqrt{x_{1} x_{2}}$. There are several choices of $V$ for which exactly solvable models can be constructed. The example of a system of coupled nonlinear oscillators is considered in this article in some detail. The potential is chosen as,

$$
V(r)=\frac{1}{2} \omega^{2} r^{2}+\frac{\alpha}{4} r^{4},
$$

which gives the following Eq. of motion,

$$
\ddot{x}_{i}-(-1)^{i+1} 2 \gamma \dot{x}_{i}+\omega^{2} x_{i}+\alpha\left(x_{1} x_{2}\right) x_{i}=0, i=1,2 .
$$

The parameter $\alpha$ controls the strength of the nonlinear interaction term, whereas $\omega$ is the angular frequency of the harmonic term. A system of uncoupled oscillators with balanced loss and gain, i.e., the system considered in Ref. [1, is obtained for $\alpha=0$. The constant of motion $\hat{L}$ has the form,

$$
\hat{L}=\left(\frac{-\gamma}{2}\right)\left(\dot{x}_{1} x_{2}-\dot{x}_{2} x_{1}-2 \gamma x_{1} x_{2}\right),
$$

which is independent of $\alpha$. The overall multiplication factor has no significance and one may define $\tilde{L}=-\frac{2}{\gamma} \hat{L}$ as the constant of motion. The expression for $\tilde{L}$ may also be obtained directly from Eq. (121). The constant value of $\hat{L}$ is determined from the initial conditions imposed on $x_{i}(0)$ and $\dot{x}_{i}(0)$. The value $\hat{L}=0$ may be obtained for different sets of boundary conditions. For $\hat{L}=0, x_{1}(t)$ and $x_{2}(t)$ are related to each other. In particular,

$$
x_{1}=c_{0} e^{\gamma t} q(t), \quad x_{2}=d_{0} e^{-\gamma t} q(t), \quad c_{0}, d_{0} \in \Re,
$$

where $q(t)$ satisfies the equation of a cubic nonlinear oscillator:

$$
\ddot{q}+\Omega^{2} q+\Gamma q^{3}, \quad \Omega^{2} \equiv\left(\omega^{2}-\gamma^{2}\right), \quad \Gamma \equiv \alpha c_{0} d_{0} .
$$

which is exactly solvable. This equation is identical to the second Eq. of (64) with the identification $\Omega^{2}=\omega^{2}, \Gamma=\alpha$. Exact non-singular solutions for $q$ can be found. However, $x_{1}$ is always a decaying solution, while $x_{2}$ is a growing solution. The system of harmonic oscillators with balanced loss and gain and without any coupling between the two has similar solutions. The introduction of a nonlinear coupling between the two as specified in Eq. (121) do not give any stable solutions. The specific type of non-linearity for which $\hat{L}$ is a constant of motion is not suitable for obtaining classically stable solutions. 


\subsubsection{Solvable Model of $N>2$}

A solvable model of $N=2 m$ numbers of coupled nonlinear oscillators are presented in this section. The matrices $M, D, R$ are given by Eq. (18) with $\eta=\gamma I_{m}$ and $\kappa=I_{m}$ and $G$ is chosen as $G=M^{-1}$ so that the variable $r$ has the following form:

$$
r^{2}=\sum_{i=1}^{m} x_{2 i-1} x_{2 i}=\sum_{i=1}^{m}\left[\left(z_{i}^{+}\right)^{2}-\left(z_{i}^{-}\right)^{2}\right] .
$$

It may be noted that $r$ has the interpretation of the radial variable in a pseudo-Euclidean coordinate with the signature of the metric as $(1,-1,1,-1, \ldots, 1,-1)$. With the choice of the potential $V \equiv V(r)$, the Hamiltonian (49) with $\gamma=0$ is rotationally invariant in this pseudoEuclidean co-ordinate. The rotational invariance of the Hamiltonian (49) is partially lost for $\gamma \neq 0$, since the term linear in $\gamma$ is the sum of angular momenta for rotations in $m$ planes specified by ' $z_{i}^{-}-z_{i}^{+}$'. The Hamiltonian (49) is invariant under rotation when the planes of rotations are chosen as ' $z_{i}^{-}-z_{i}^{+}$'

The Eqs. of motion for a $V \equiv V(r)$ with $r$ given by Eq. (125) reads,

$$
\ddot{z}_{i}^{+}-2 \gamma \dot{z}_{i}^{-}+\frac{1}{r} \frac{\partial V}{\partial r} z_{i}^{+}=0, \ddot{z}_{i}^{-}-2 \gamma \dot{z}_{i}^{+}+\frac{1}{r} \frac{\partial V}{\partial r} z_{i}^{-}=0 i=1,2, \ldots m .
$$

Multiplying the Eq. for $z_{i}^{+}$by $\dot{z}_{i}^{-}$, Eq. for $z_{i}^{-}$by $\dot{z}_{i}^{+}$and subtracting the resulting equations, $m$ constants of motion may be obtained as,

$$
\hat{L}_{i}=\dot{z}_{i}^{+} z_{i}^{-}-z_{i}^{+} \dot{z}_{i}^{-}+\gamma\left[\left(z_{i}^{+}\right)^{2}-\left(z_{i}^{-}\right)^{2}\right], i=1,2, \ldots, m .
$$

These constants of motion along with the Hamiltonian constitute $m+1$ number of integrals of motion,

$$
\left\{\hat{L}_{i}, \hat{L}_{j}\right\}_{P B}=0,\left\{H, \hat{L}_{i}\right\}_{P B}=0,
$$

implying that the system at least partially integrable. The co-ordinates may be parametrized in terms of $m$ functions $q_{i}(t)$ as,

$$
z_{i}^{+}(t)=q_{i}(t) \cosh (\gamma t), z_{i}^{-}(t)=q_{i}(t) \sinh (\gamma t),
$$

for which the values of all the constants of motion are zero, i,e, $\hat{L}_{i}=0, \forall i$ and $r^{2}=\sum_{i=1}^{m} q_{i}^{2} \equiv q^{2}$. With the substitution of $z_{i}^{ \pm}(t)$ in the equations of motion (126), the following equations in terms of $q_{i}$ 's are obtained:

$$
\ddot{q}_{i}-\gamma^{2} q_{i}+\frac{1}{q} \frac{\partial V(q)}{\partial q} q_{i}=0, i=1,2, \ldots, m .
$$

Exactly solvable models may be constructed for suitable choices of $q$.

A particular example of exactly solvable model is a chain of coupled nonlinear oscillators. The equation (130), with the choice of the potential as in (120) and the radial variable defined by (125), can be expressed as,

$$
\ddot{q}_{i}+\Omega^{2} q_{i}+\alpha q^{2} q_{i}=0, \Omega^{2}=\omega^{2}-\gamma^{2} .
$$

which is exactly solvable. In terms of $r$ in Eq. (125), Eq. (131) takes the form of Eq. (83) with $\delta=0$ and $\omega$ replaced by $\Omega$. Therefore, with this identification of parameters the solutions of Eq. (83) are also valid solutions of Eq. (131). The solutions of $x_{2 i}$ are always a decaying one, while that of $x_{2 i-1}$ are a growing one. The system of harmonic oscillators with balanced loss and gain and without any coupling between them has similar solutions. The introduction of a nonlinear coupling as in (120) does not produce any stable solutions. 


\section{Summary \& Discussions}

A Hamiltonian formulation of a generic many-body system with balanced loss and gain has been investigated. The form of a generic many-particle Hamiltonian including gauge potential has been assumed. The requirement that the resulting Eqs. of motion contain balanced loss and gain terms severely restricts the allowed range of the gain-loss coefficient and the possible form of the interacting potential. The balancing of the loss and gain terms occur in a pair-wise fashion, i.e corresponding to the coefficient $\gamma$ of a loss term there exits necessarily a gain term having the coefficient $-\gamma$ and balancing of $\gamma$ by two of more terms are not allowed. It has been found that some of the well-known many-particle systems like Calogero models are not amenable to Hamiltonian formulation for three or more particles, if they are generalized to include balanced loss and gain terms. In the Calogero model each particle interacts with rest of the particles which is manifested in the respective equations of motion. If this feature is sacrificed, then a Hamiltonian formulation for many-body system having inverse square potential plus a harmonic term may be incorporated in the context where loss and gain are balanced. In fact, in spite of the imposing restriction, the Hamiltonian formulation for a large class of system is formulated with balancing loss and gain terms that also includes some of the known examples. Finally, it is shown that the Hamiltonian can always be reformulated in the background of a pseudo-Euclidean metric through some transformations of the coordinate. A Hamiltonian formulation for systems with space-dependent balanced loss and gain terms is also presented. The system having balanced loss and gain is investigated from the viewpoint of exactly solvable models. Two types of exactly solvable models with balanced loss and gain are considered. Type-I system is characterized by a potential which has a translational symmetry although the Hamiltonian does not respect this symmetry. The type-II system is characterized by a potential which has a rotational symmetry in the background of a space having pseudo-Euclidean metric of the form $g_{i j}=(-1)^{i+1} \delta_{i j}$. The Hamiltonian for type-II models is not invariant under rotation in any plane, but, invariant under rotations in specific planes. For both types of systems, apart from the Hamiltonian, $m$ number of integrals of motion are constructed where $N=2 m$ is the number of particles in the system. These integrals of motion are in involution implying that the system is at least partially integrable. The existence of $m$ degrees of freedom make it possible to determine the dynamics of the original system with $2 m$ degrees of freedom in terms of an effective system having $m$ degrees of freedom. The exactly solvability of the effective system ensures the same for the original Hamiltonian.

For the type-I models, stable classical solutions are obtained in terms of Jacobi elliptic functions for particular ranges of parameters. For the case of a single quartic nonlinear oscillator, stable solutions are obtained even if the gain-loss parameter $\gamma$ is varied without any upper bound. Exact solutions are obtained in closed analytical form for several models which are appropriate generalizations of well-known systems like, two particles coupled nonlinear oscillators, Henon-Heils system, Calogero-type model etc. Some exactly solvable many particle systems with balanced loss and gain and are interacting via a four-body potential are investigated. The exact solutions of these model are obtained by exploiting the known solutions of Calogero-type of models. It is found that the balancing of the loss and gain terms in a pair-wise fashion highly restricts the possible form of the four-body interaction. Exact solutions are obtained for several type-II models, including coupled chain of nonlinear oscillators. However, the solutions are not stable and there exists no region in the parameter-space for which stable solutions are possible. There are equal number of growing and decaying solutions for even number of particles. Exact solutions are obtained in terms of Jacobi elliptic functions multiplied by an exponentially decaying/growing factor.

The Schwinger-Keldysh formalism is an useful tool for studying nonequlibrium many-particle systems. It is being used in a variety of contemporary topics like driven open quantum systems 50 , 
[51. 52, time-dependent density-functional theory [53], relativistic hydrodynamics, physics of black-holes, dynamics of entanglement in quantum field theory etc. 54. The invariance of Schwinger-Keldysh action under time-reversal symmetry plus time-translation corresponds to thermodynamic equilibrium [51]. The Hamiltonian is the generator of the time-translation and unitary time evolution, while invariance under time-reversal symmetry conforms to principle of detailed balance.

The above result is worth comparing with that of the systems with balanced loss and gain, where an equilibrium is reached in regard to energy transfer between the system and the bath for unbroken $\mathcal{P} \mathcal{T}$ symmetry [25, 31, 32. The action corresponding to the Lagrangian in Eq. (6) and the associated Hamiltonian are invariant under time-translation, as in the case of SchwingerKeldysh action at thermodynamic equilibrium. An apparent difference between the two cases arises in respect to discrete symmetries. The Schwinger-Keldysh action at thermodynamic equilibrium is invariant under time-reversal symmetry, whereas unbroken $\mathcal{P} \mathcal{T}$ symmetry is essential for the existence of stable classical solutions of $H$ in Eq. (3) or the respective quantum bound states 25, 31, 32, 55. This apparent difference may be removed, if a non-conventional time-reversal symmetry [56] is used instead of the conventional one, which generates the same canonical transformations as in the case of $\mathcal{P} \mathcal{T}$ symmetry. For example, the Hamiltonian in Eq. (49) can be interpreted as a system of $m$ particles on the two dimensional plane embedded in a three dimensional system. Following Ref. [56], a non-conventional time-reversal $\operatorname{symmetry}(\hat{\mathcal{T}})$ for the many-particle system may be defined as,

$$
\begin{aligned}
& \hat{\mathcal{T}}=\exp \left(i \pi \sum_{i=1}^{m} J_{z_{i}^{-}}\right) \mathcal{T} \\
& \hat{\mathcal{T}}: z_{i}^{+} \rightarrow-z_{i}^{+}, z_{i}^{-} \rightarrow z_{i}^{-}, P_{z_{i}^{+}} \rightarrow P_{z_{i}^{+}}, P_{z_{i}^{-}} \rightarrow-P_{z_{i}^{-}},
\end{aligned}
$$

where $J_{z_{i}^{-}}$denotes generator of rotation for the $i$-th particle around $z^{-}$-axis and $\mathcal{T}$ is the conventional time-reversal operator. If the third degree of freedom for the $i$-th particle is denoted as $z_{i}^{0}$, then $\hat{\mathcal{T}}: z_{i}^{0} \rightarrow-z_{i}^{0}, P_{z_{i}^{0}} \rightarrow P_{z_{i}^{0}}$ ensures that $\hat{\mathcal{T}} \mathcal{T}^{-1}$ describes a proper rotation in three dimensional space. Further, for any two quantum mechanical states $|\psi\rangle$ and $|\phi\rangle,\langle\hat{\mathcal{T}} \phi \mid \hat{\mathcal{T}} \psi\rangle=\langle\psi \mid \phi\rangle$ signifies time-reversal invariance. Thus, the $\mathcal{P} \mathcal{T}$ symmetry on the plane and the non-conventional time-reversal symmetry $\hat{\mathcal{T}}$ in three dimensions induces the same canonical transformations on the $z^{-}-z^{+}$plane. Consequently, the conditions for thermodynamic equilibrium of the SchwingerKeldysh action and equilibrium condition for systems with balanced loss and gain may be identified as similar.

Some future directions of study may be listed as follows:

- The many particle interaction of Calogero model is governed by root system of finite reflection groups. The type of Calogero model considered in this work belongs to A-type root system. However, Calogero-Moser system is integrable for other root systems such as B,C,D and also for the exceptional and non-crystallographic root systems. Therefore, the investigation of integrability and exact solvability of all such root systems in the presence of the balanced loss and gain terms will be a part of the future studies. Further, the integrability and exact solvability of impenetrable system where the nearest neighbour and the next-to-nearest neighbours interact[57] in the presence of the balanced loss and gain terms will also be a part of the future investigation.

- The field equations arising from the large $\mathrm{N}$ limit of all such systems are to be investigated (See, for example, 58, and the references therein). 
- In the pseudo-euclidean metrics Chern-Simons gauge theory in the infrared region is associated with dissipative dynamics 38 . The connection of the balanced loss and gain systems considered in this work with particular gauge theory will be very much interesting.

- In the present work, we mainly concerned with the Hamiltonian formulation of a generic many-body systems with balanced loss and gain, their integrability and exact solvability at the classical level. The quantization of this kind of systems will be a part of the future investigation.

\section{Acknowledgements}

This work is partly supported by a grant(DST Ref. No. SR/S2/HEP-24/2012) from Science \& Engineering Research Board(SERB), Department of Science \& Technology(DST), Govt. of India. DS acknowledges a research fellowship from CSIR.

\section{Appendix A}

The choices of $V$ are ubiquitous for which the system governed by Eq. (60) is exactly solvable. In this appendix, results of a simple exactly solvable potentials, i.e solution for Henon-Heils system [59] is presented. This system was first time introduced by Henon and Heils in 1964 while examining the constants of motion in galactic dynamics. The system is characterized by the potential

$$
V=\sum_{i=1}^{2}\left(-\omega_{i}^{2} z_{i}^{2}\right)-\frac{\alpha}{2} z_{1}^{2} z_{2}-\frac{1}{6} \beta z_{2}^{3} .
$$

There are three distinct regions in parameter-space for which exact solutions exist 44]. For example, for the choice of the parameters $\omega_{1}=\omega_{2} \equiv \omega_{0}, \beta=-\alpha, z_{i}$ satisfy the equation:

$$
\ddot{z}_{1}+\omega^{2} z_{1}+2 \alpha z_{1} z_{2}=0, \ddot{z}_{2}+\omega^{2} z_{2}+\alpha\left(z_{1}^{2}+z_{2}^{2}\right)=0, \omega^{2}=4\left(\omega_{0}^{2}-\gamma^{2}\right) .
$$

These two equations are separable in the co-ordinate, $u=z_{1}+z_{2}, v=z_{1}-z_{2}$ and describe two oscillators with quadratic interaction:

$$
\ddot{u}+\omega^{2} u+\alpha u^{2}=0, \ddot{v}+\omega^{2} v+\alpha v^{2}=0 .
$$

These Eqs. are exactly solvable with the solutions having the form [60]:

$$
\begin{gathered}
u_{i}=A_{i} c n^{2}\left[\Omega_{i} t, k_{i}^{2}\right]+b_{i}, k_{i}^{2}=\frac{A_{i}^{2} \alpha}{6 \Omega_{i}^{2}}, b_{i}=\frac{-4\left[\Omega_{i}^{2}\left(2 k_{i}^{2}-1\right)+\omega^{2}\right]}{2 \alpha}, \quad 0<k_{i}<1, \\
\Omega_{i}^{4}=\frac{\omega^{4}}{16\left(k_{i}^{4}-k_{i}^{2}+1\right)}, \quad i=1,2, \quad u_{1}=u, u_{2}=v
\end{gathered}
$$

and

$$
\begin{gathered}
z_{1}=\frac{1}{2}[u+v], \quad z_{2}=\frac{1}{2}[u-v] . \\
z_{1}^{+}=\gamma\left[A_{1} E\left(k_{1}, A_{1}\right)+A_{2} E\left(k_{2}, A_{2}\right)\right], \quad z_{2}^{+}=\gamma\left[A_{1} E\left(k_{2}, A_{2}\right)-A_{2} E\left(k_{2}, A_{2}\right)\right],
\end{gathered}
$$


where

$$
E\left(k_{i}, A_{i}\right)=\left(t-\frac{t}{k_{i}^{2}}\right)+\frac{\text { EllipticalE }\left[a m\left[\Omega_{i} t, k_{i}^{2}\right], k^{2}\right]\left(\mathrm{cn}^{2}\left[\Omega_{i} t, k_{i}^{2}\right]+\frac{1}{k_{i}^{2}}-1\right)}{\Omega_{i} d n\left[\Omega_{i} t, k_{i}^{2}\right] \sqrt{1-k_{i}^{2} s n^{2}\left[\Omega_{i} t, k_{i}^{2}\right]}} .
$$

It should be mentioned here that the expression of $E(t)$ encounters singularity and therefore the solutions of $z_{i}^{+}$'s are not stable.

\section{Appendix B}

The Lagrangian of a $\mathrm{N}$ particle rotationally symmetric system in hyper-spherical coordinates has the following form:

$$
L=\frac{1}{2}\left[\dot{r}^{2}+r^{2} \dot{\theta}_{1}^{2}+r^{2}\left\{\sum_{i=2}^{N-1}\left(\dot{\theta}_{i}^{2} \prod_{j=1}^{i-1} \sin ^{2} \theta_{j}\right)\right\}\right]-V(r) .
$$

The corresponding Hamiltonian is:

$$
H=\frac{1}{2}\left[p_{r}^{2}+\frac{p_{\theta_{1}}^{2}}{r^{2}}+\sum_{i=2}^{N-1} \frac{p_{\theta_{i}}^{2}}{r^{2} \prod_{j=1}^{i-1} \sin ^{2} \theta_{j}}\right]+V(r)
$$

The Hamilton-Jacobi (HJ) characteristic function in this case may be written as:

$$
W=W_{r}(r)+\sum_{i=1}^{N-1} W_{\theta_{i}}\left(\theta_{i}\right)+\alpha_{N-2} \theta_{N-1}
$$

with $\theta_{N-1}$ being a cyclic coordinate. The (HJ) equation takes the following form:

$$
\left[\left(\frac{\partial W_{r}}{\partial r}\right)^{2}+\frac{1}{r^{2}}\left\{\left(\frac{\partial W_{\theta_{1}}}{\partial \theta_{1}}\right)^{2}+\sum_{i=2}^{N-1} \frac{1}{\prod_{j=1}^{i-1} \sin ^{2} \theta_{j}}\left(\frac{\partial W_{\theta_{i}}}{\partial \theta_{i}}\right)^{2}\right\}\right]+2 V(r)=2 E .
$$

Now, the term in the curly bracket is only a function of the angular variables and must therefore be a constant $\left(=\alpha_{r}^{2}\right)$. From Eq. (143), we therefore have:

$$
\begin{aligned}
& \left(\frac{\partial W_{r}}{\partial r}\right)^{2}+\frac{\alpha_{r}^{2}}{r^{2}}=2(E-V) \\
& \left(\frac{\partial W_{\theta_{1}}}{\partial \theta_{1}}\right)^{2}+\sum_{i=2}^{N-1} \frac{1}{\prod_{j=1}^{i-1} \sin ^{2} \theta_{j}}\left(\frac{\partial W_{\theta_{i}}}{\partial \theta_{i}}\right)^{2}=\alpha_{r}^{2}
\end{aligned}
$$

The first Eq. (144) gives

$$
W_{r}=\int \sqrt{2(E-V)-\frac{\alpha_{r}^{2}}{r^{2}}} d r
$$

and the second Eq. (145) may be written as:

$$
\left(\frac{\partial W_{\theta_{1}}}{\partial \theta_{1}}\right)^{2}+\frac{1}{\sin _{\theta_{1}}^{2}}\left\{\left(\frac{\partial W_{\theta_{2}}}{\partial \theta_{2}}\right)^{2}+\sum_{i=3}^{N-1} \frac{1}{\prod_{j=2}^{i-1} \sin ^{2} \theta_{j}}\left(\frac{\partial W_{\theta_{i}}}{\partial \theta_{i}}\right)^{2}\right\}=\alpha_{r}^{2},
$$


again the term in the curly bracket is devoid of $\theta_{1}$ and must be a constant $\alpha_{\theta_{1}}^{2}$. Thus from Eq. (147), we have

$$
W_{\theta_{1}}=\int \sqrt{\alpha_{r}^{2}-\frac{\alpha_{\theta_{1}^{2}}}{\sin ^{2} \theta_{1}}} d \theta_{1} .
$$

By carrying on similar procedure we have in general:

$$
W_{\theta_{i}}=\int \sqrt{\alpha_{i-1}^{2}-\frac{\alpha_{\theta_{i}^{2}}}{\sin ^{2} \theta_{i}}} d \theta_{i} .
$$

Thus if $\beta_{r}, \beta_{\theta_{i}} \forall i, i=1 \ldots N-1$ are the initial values of the radial and angular coordinates respectively then from Eq. (142) we have:

$$
\begin{aligned}
& \beta_{t}+t=\frac{\partial W}{\partial E}=\int \frac{d r}{\sqrt{2(E-V)-\frac{\alpha_{r}^{2}}{r^{2}}}}, \\
& \beta_{r}=\frac{\partial W}{\partial \alpha_{r}}=-\int \frac{\alpha_{r} d r}{r^{2} \sqrt{2(E-V)-\frac{\alpha_{r}^{2}}{r^{2}}}}+\int \frac{\alpha_{r} d \theta_{1}}{\sqrt{\alpha_{r}^{2}-\frac{\alpha_{\theta_{1}}^{2}}{\sin ^{2} \theta_{1}}}}, \\
& \beta_{\theta_{i}}=\frac{\partial W}{\partial \alpha_{\theta_{i}}}=-\int \frac{\alpha_{\theta_{i}} d \theta_{i}}{\sin ^{2} \theta_{i} \sqrt{\alpha_{\theta_{i-1}}^{2}-\frac{\alpha_{\theta_{i}}^{2}}{\sin ^{2} \theta_{i}}}}+\int \frac{\alpha_{\theta_{i}} d \theta_{i+1}}{\sqrt{\alpha_{\theta_{i}}^{2}-\frac{\alpha_{\theta_{i+1}}^{2}}{\sin ^{2} \theta_{i+1}}}},
\end{aligned}
$$

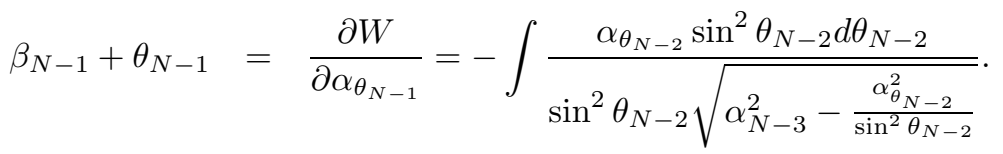

Thus, we have $n+1$ integral Eqs. The first Eq. gives $r$ as function of time. The differential form of which may be written as:

$$
\ddot{r}-\frac{\alpha_{r}^{2}}{r^{3}}+\frac{\partial V}{\partial r}=0
$$

which can be solved for the specific form of the potential $V$. It may be noted that in order to map Eq. (151) to that of considered in Sec. 3.1.2, one needs to replace $V$ by $-V$. The second Eq. gives the relation between $r$ and $\theta_{1}$ and may be written as

$$
\beta_{r}=\frac{\partial W}{\partial \alpha_{r}}=-\int \frac{\alpha_{r} d r}{r^{2} \sqrt{2(E-V)-\frac{\alpha_{r}^{2}}{r^{2}}}}-\sin ^{-1}\left(\frac{\alpha_{r}}{\sqrt{\alpha_{r}^{2}-\alpha_{\theta_{1}}^{2}}} \cos \theta_{1}\right) .
$$

Rest of the Eqs. give relations between $\theta_{i}$ and $\theta_{i+1} \forall i, i=2, \ldots . . N-2$. Thus, once the solutions of the set of Eqs. in (150) are known, the radial and angular variables can be expressed as a function of time.

\section{Appendix $\mathrm{C}$}

The Lax-pair for the system (99) may be written as 48]:

$$
L_{i j}=p_{z_{i}} \delta i j+\left(1-\delta_{i j}\right) \frac{i g}{\sqrt{2}\left(z_{i}-z_{j}\right)},
$$




$$
\mathcal{M}_{i j}=\frac{i g}{\sqrt{2}}\left[\delta_{i j} \sum_{l,(l \neq i)}^{m} \frac{1}{\left(z_{i}-z_{l}\right)^{2}}-\left(1-\delta_{i j}\right) \frac{1}{\left(z_{i}-z_{j}\right)^{2}}\right]
$$

The diagonal matrix $X(t)$ is defined by $X(t)=\delta_{i j} z_{i}(t)$. The Eq. (99) may now be written in the following matrix form:

$$
\dot{X}-[X, \mathcal{M}]=L, \quad \dot{L}-[L, \mathcal{M}]=-\omega^{2} X
$$

We define another matrix $Q(t)$ in the following fashion:

$$
Q(t)=U(t) X(t) U^{-1}(t),
$$

where $U(t)$ is a unitary matrix, satisfying the relation:

$$
\dot{U}=U \mathcal{M}, \quad U(0)=1_{m \times m} .
$$

With this constructions it is easy to evaluate that

$$
\begin{aligned}
& \dot{Q}=U(\dot{X}-[X, \mathcal{M}]) U^{-1}=U L U^{-1}, \\
& \ddot{Q}=U(\dot{L}-[L, \mathcal{M}]) U^{-1}=-\omega^{2} Q
\end{aligned}
$$

with the following solutions:

$$
Q(t)=Q(0) \cos (\omega t)+\omega^{-1} \dot{Q}(0) \sin (\omega t),
$$

where $Q(0)=X(0)$ and $\dot{Q}(0)=L(0)$ are obtained from the initial values of $z_{i}(0)$ and $p_{z_{i}}(0)$.

\section{Appendix D: $D_{N}$ type Calogero system with four-body interaction}

$D_{N}$ type Calogero system arises if we take the potential $V\left(z_{i}\right)$ as

$$
V\left(z_{i}\right)=-\sum_{i}^{m} 2 \omega_{0}^{2} z_{i}^{2}-\sum_{\substack{i, j=1 \\ i<j}}^{m} \frac{g^{2}}{2\left(z_{i}-z_{j}\right)^{2}}-\sum_{\substack{i, j=1 \\ i<j}}^{m} \frac{g^{2}}{2\left(z_{i}+z_{j}\right)^{2}}
$$

and the Eq. of motion (60) becomes:

$$
\begin{aligned}
& \ddot{z}_{i}+\omega^{2} z_{i}-\sum_{j,(j \neq i)}^{m}\left[\frac{g^{2}}{\left(z_{i}-z_{j}\right)^{3}}+\frac{g^{2}}{\left(z_{i}+z_{j}\right)^{3}}\right]=0, \\
& z_{i}^{+}(t)=2 \gamma \int z_{i}(t) d t+C_{i}, \quad i=1,2, \ldots m .
\end{aligned}
$$

The solution of Eq. (162) may be obtained from the Lax-pair formulation [48, 61]. The Lax-pair for the system (162) may be written as:

$$
\mathbf{L}=\left(\begin{array}{cc}
L & S \\
-S & -L
\end{array}\right), \quad \mathbf{M}=\left(\begin{array}{cc}
\mathcal{M} & T \\
T & \mathcal{M}
\end{array}\right), \quad \mathbf{X}=\left(\begin{array}{cc}
X & 0 \\
0 & -X
\end{array}\right)
$$


where

$$
\begin{aligned}
L_{i j} & =p_{z_{i}} \delta i j+\left(1-\delta_{i j}\right) \frac{i g}{\sqrt{2}\left(z_{i}-z_{j}\right)} \\
\mathcal{M}_{i j} & =\frac{1}{\sqrt{2}}\left[\delta_{i j} \sum_{l,(l \neq i)}^{m}\left[\frac{i g}{\left(z_{i}-z_{l}\right)^{2}}+\frac{i g}{\left(z_{i}+z_{l}\right)^{2}}\right]-\left(1-\delta_{i j}\right) \frac{i g}{\left(z_{i}-z_{j}\right)^{2}}\right] \\
S_{i j} & =\left(1-\delta_{i j}\right) \frac{i g}{\sqrt{2}\left(z_{i}+z_{j}\right)} \\
T_{i j} & =-\left(1-\delta_{i j}\right) \frac{i g}{\sqrt{2}\left(z_{i}+z_{j}\right)^{2}}
\end{aligned}
$$

and the diagonal matrix $X(t)$ is defined by $X(t)=\delta_{i j} z_{i}(t)$. The Eq. (162) may now be written in the following matrix form:

$$
\dot{\mathbf{X}}-[\mathbf{X}, \mathbf{M}]=\mathbf{L}, \quad \dot{\mathbf{L}}-[\mathbf{L}, \mathbf{M}]=-\omega^{2} \mathbf{X}
$$

We define another matrix $Q(t)$ in the following fashion:

$$
Q(t)=U(t) \mathbf{X}(t) U^{-1}(t),
$$

where $U(t)$ is a unitary matrix, satisfying the relation:

$$
\dot{U}=U \mathbf{M}, \quad U(0)=1_{m \times m} .
$$

With this constructions it is easy to evaluate that

$$
\begin{aligned}
& \dot{Q}=U(\dot{\mathbf{X}}-[\mathbf{X}, \mathbf{M}]) U^{-1}=U \mathbf{L} U^{-1} \\
& \ddot{Q}=U(\dot{\mathbf{L}}-[\mathbf{L}, \mathbf{M}]) U^{-1}=-\omega^{2} Q
\end{aligned}
$$

with the following solutions:

$$
Q(t)=Q(0) \cos (\omega t)+\omega^{-1} \dot{Q}(0) \sin (\omega t),
$$

where $Q(0)=X(0)$ and $\dot{Q}(0)=L(0)$ are obtained from the initial values of $z_{i}(0)$ and $p_{z_{i}}(0)$. Thus, $z_{i}$ 's are obtained from the eigen values of the matrix $Q(t)$ and $z_{i}^{+}$'s are obtained from the eigen values of the matrix $R(t)$ :

$$
R(t)=\frac{Q(0)}{\omega} \sin (\omega t)-\omega^{-2} \dot{Q}(0) \cos (\omega t)
$$

Once the eigen values of the matrices $Q(t)$ and $R(t)$ are known, $x_{2 i-1}$ and $x_{2 i}$ can easily be constructed using Eq. (48). After a coordinate transformation of the form (48), the Eq. (162) gives rise to the following Eqs. of motion:

$$
\begin{aligned}
& \ddot{x}_{2 l-1}+2 \omega_{0}^{2}\left(x_{2 l-1}-x_{2 l}\right)-2 \gamma \dot{x}_{2 l-1} \\
- & \sum_{\substack{i=1 \\
i \neq l}}^{m} 2 g^{2}\left[\frac{1}{\left(x_{2 i-1}-x_{2 i}-x_{2 l-1}+x_{2 l}\right)^{3}}+\frac{1}{\left(x_{2 i-1}-x_{2 i}+x_{2 l-1}-x_{2 l}\right)^{3}}\right]=0, \\
& \ddot{x}_{2 l}+2 \gamma \dot{x}_{2 l}-2 \omega_{0}^{2}\left(x_{2 l-1}-x_{2 l}\right)
\end{aligned}
$$




$$
\begin{aligned}
& +\sum_{\substack{i=1 \\
i \neq l}}^{m} 2 g^{2}\left[\frac{1}{\left(x_{2 i-1}-x_{2 i}-x_{2 l-1}+x_{2 l}\right)^{3}}+\frac{1}{\left(x_{2 i-1}-x_{2 i}+x_{2 l-1}-x_{2 l}\right)^{3}}\right]=0, \\
& \quad l=1, . ., m
\end{aligned}
$$

with

$V=-\sum_{i}^{m} \omega_{0}^{2}\left(x_{2 i-1}-x_{2 i}\right)^{2}-\sum_{\substack{i, j=1 \\ i<j}}^{m} g^{2}\left[\frac{1}{\left(x_{2 i-1}-x_{2 i}-x_{2 j-1}+x_{2 j}\right)^{2}}+\frac{1}{\left(x_{2 i-1}-x_{2 i}+x_{2 j-1}-x_{2 j}\right)^{2}}\right]$.

\section{References}

[1] H. Bateman, Phys. Rev. 38, 815 (1931).

[2] P. M. Morse and H. Feshbach, Methods of Theoretical Physics (McGraw-Hill, New York, 1953), Vol. I.

[3] F. Bopp, Sitz.-Bcr. Bayer. Akad. Wiss. Math.-naturw. KI. 67, (1973).

[4] H. Feshbach and Y. Tikochinsky, in A Festschrift for I. I. Rabi, Trans. New York. Acad. Sci., Series 2 38, 44 (1977).

[5] Y. Tikochinsky, J. Math. Phys. 19, 888 (1978).

[6] H. Dekker, Phys. Rep. 80, 1 (1981).

[7] E. Celeghini, M. Rasetti, and G. Vitiello, Ann. Phys. (N.Y) 215, 156 (1992).

[8] R. Banerjee and P. Mukherjee, J. Phys. A: Math. Gen. 35, 5591 (2002).

[9] D. Chruscinski and J. Jurkowski, Ann. Phys. (N.Y.) 321, 854 (2006).

[10] C. M. Bender and S. Boettcher, Phys. Rev. Lett. 80, 5243 (1998); C. M. Bender, Contemp. Phys. 46, 277 (2005).

[11] A. Mostafazadeh, Int. J. Geom. Methods Mod. Phys. 7, 1191 (2010); J. Math. Phys. 43, 205 (2002); 43, 2814 (2002); 43, 3944 (2002); Nucl. Phys. B 640, 419 (2002).

[12] P. K. Ghosh, J. Phys.: Condens. Matter 24, 145302 (2012); Phys. Lett. A 375, 3250 (2011); J. Phys. A 44, 215307 (2011); Int. J. Theor. Phys. 50, 1143 (2011); J. Phys. A 43, 125203 (2010).

[13] A. Fring, Philos. Trans. R. Soc. London, Ser. A 371, 20120046 (2013).

[14] M. Znojil, Ann. Phys. (NY) 336, 98 (2013); Phys. Lett. B 647, 225 (2007).

[15] J. Rubenstein, P. Sternberg, and Q. Ma, Phys. Rev. Lett. 99, 167003 (2007).

[16] N. M. Chtchelkatchev, A. A. Golubov, T. I. Baturina, and V. M. Vinokur, Phys. Rev. Lett. 109, 150405 (2012).

[17] A. Guo, G. J. Salamo, D. Duchesne, R. Morandotti, M. Volatier-Ravat, V. Aimez, G. A. Siviloglou, and D. N. Christodoulides, Phys. Rev. Lett. 103, 093902 (2009). 
[18] C. E. Ruter, K. G. Makris, R. El-Ganainy, D. N. Christodoulides, M. Segev, and D. Kip, Nature Physics 6, 192 (2010).

[19] A. Regensburger, M. A. Miri, C. Bersch, J. Nager, G. Onishchukov, D. N. Christodoulides, and U. Peschel, Phys. Rev. Lett. 110, 223902 (2013).

[20] V. V. Konotop, J. Yang, D. A. Zezyulin, Rev. Mod. Phys. 88, 035002 (2016).

[21] S. Bittner, B. Dietz, U. Gunther, H. L. Harney, M. Miski-Oglu, A. Richter, and F. Schfer, Phys. Rev. Lett. 108, 024101 (2012).

[22] K. F. Zhao, M. Schaden, and Z. Wu, Phys. Rev. A 81, 042903 (2010).

[23] C. Zheng, L. Hao, G. L. Long, Phil. Trans. R. Soc. A 371, 20120053 (2013).

[24] J. Schindler, A. Li, M. C. Zheng, F. M. Ellis, and T. Kottos, Phys. Rev. A 84, 040101(R) (2011).

[25] C. M. Bender, M. Gianfreda, S. K. Ozdemir, B. Peng, and L. Yang, Phys. Rev. A 88, 062111 (2013).

[26] B. Peng, S. K. Ozdemir, F. Lei, F. Monifi, M. Gianfreda, G. L. Long, S. Fan, F. Nori, C. M. Bender, and L. Yang, Nature Physics 10, 394 (2014).

[27] A. O. Caldeira and A. J. Leggett, Phys. Rev. Lett. 46, 211 (1981).

[28] N. Lang and H. P. Buchler, Phys. Rev. A 92, 012128 (1015).

[29] J. Cuevas, P. G. Kevrekidis, A. Saxena, and A. Khare, Phys. Rev. A 88, 032108 (2013).

[30] I. V. Barashenkov and M. Gianfreda, J. Phys. A: Math. Theor. 47, 282001(2014).

[31] C. M. Bender, M. Gianfreda, and S. P. Klevansky Phys. Rev. A 90, 022114 (2014).

[32] D. Sinha, P. K. Ghosh, arXiv:1705:03426.

[33] F. Calogero, Jour. Math. Phys. 10, 2191 (1969), F. Calogero, Jour. Math. Phys. 10, 2197 (1969), F. Calogero, Jour. Math. Phys. 12, 419 (1971).

[34] B. Sutherland, J. Math. Phys.(N.Y.)12, 246 (1971); 12, 251 (1971); Phys.Rev. A 4, 2019 (1971).

[35] M. A. Olshanetsky and A. M. Perelomov, Phys. Rep. 71, 314 (1981); 94, 6 (1983).

[36] A. P. Polychronakos, Phys. Rev. Lett. 69, 703 (1992).

[37] P. K. Ghosh, J. Phys. A: Math. Theor. 45, 183001 (2012).

[38] M. Blasone, E. Graziano, O. K. Pashaev, G. Vitiello, Annals Phys. 252, 115-132 (1996).

[39] A. Pais and G.E. Uhlenbeck, Phys. Rev. 79, 145 (1950).

[40] B. Van der Pol, Philos. Mag. 43, 700 (1927).

[41] A. Venkatesan, M. Lakshmanan, Phys. Rev. E 56 (6), 63216330 (1997).

[42] D. Sinha, P. K. Ghosh (under preparation). 
[43] A. J. Bordner, E. Corrigan, and R. Sasaki, Prog. Theor. Phys. 102, 499-529 (1999).

[44] M. Lakshmanan and R. Sahadevan, Phys. Rep. 224, 1 (1993).

[45] Wolfes, Ann. Phys. 85, 454 (1974).

[46] O.Haschke, W. Ruhl, arXiv:hep-th/9807194.

[47] A. Bachkhaznadji, M. Lassaut, Few-Body Syst. 54, 1945 (2013).

[48] R. Sasaki, K. Takasaki, J.Math.Phys. 47, 012701 (2006).

[49] R. Coquereaux, Phys. Lett. B 115, 389 (1982).

[50] L. M. Sieberer, M. Buchhold, S. Diehl, Rep. Prog. Phys. 79, 096001 (2016), arXiv:1512.00637.

[51] L. M. Sieberer, A. Chiocchetta, A. Gambassi, U. C. Tuber, S. Diehl, Phys. Rev. B 92,134307 (2015), arXiv:1505.00912; L. M. Sieberer, S. D. Huber, E. Altman, S. Diehl, Phys. Rev. B 89, 134310 (2014).

[52] C. Aron, G. Biroli, and L. F. Cugliandolo, J. Stat. Mech. 1011, P11018, (2010), arXiv:1007.5059.

[53] R. van Leeuwen, N. E. Dahlen, G. Stefanucci, C. -O. Almbladh, U. von Barth, arXiv:cond-mat/0506130.

[54] F. M. Haehl, R. Loganayagam and M. Rangamani, J. High. Energ. Phys. 2017:69 (2017), arXiv:1610.01940; F. M. Haehl, R. Loganayagam, and M. Rangamani, arXiv:1610.01941.

[55] D. Sinha and P. K. Ghosh, arXive:1709.09648.

[56] F. Haake, Quantum Signatures of Chaos, Springer Series in Synergetics( 3rd edition).

[57] S. R. Jain, A. Khare, Phys. Lett. A 262, 35-39 (1999).

[58] V. Bardek, S. Meljanac, Europhys. Lett. 72, 14 (2005).

[59] M. Henon and C. Heiles, Astron. J. 69, 73 (1964).

[60] A. M. El-Naggar, G. M. Ismail, Math. Sci. Lett. 4, 313-317 (2015).

[61] T.Yamamoto, Phys. Lett. A 208, 293 (1995). 